\title{
Thermodynamically consistent phase field approach to dislocation evolution at small and large strains
}

\author{
Valery I. Levitas ${ }^{a, *}$ and Mahdi Javanbakht ${ }^{b, c}$ \\ ${ }^{a}$ Iowa State University, Departments of Aerospace Engineering, Mechanical Engineering, and \\ Material Science and Engineering, Ames, Iowa 50011, USA \\ ${ }^{b}$ Isfahan University of Technology, Department of Mechanical Engineering, Isfahan, Iran \\ ${ }^{c}$ Iowa State University, Department of Aerospace Engineering, Ames, Iowa 50011, USA
}

A thermodynamically consistent, large strain phase field approach to dislocation nucleation and evolution at the nanoscale is developed. Each dislocation is defined by an order parameter, which determines the magnitude of the Burgers vector for the given slip planes and directions. The kinematics is based on the multiplicative decomposition of the deformation gradient into elastic and plastic contributions. The relationship between the rates of the plastic deformation gradient and the order parameters is consistent with phenomenological crystal plasticity. Thermodynamic and stability conditions for homogeneous states are formulated and satisfied by the proper choice of the Helmholtz free energy and the order parameter dependence on the Burgers vector. They allow us to reproduce desired lattice instability conditions and a stress - order parameter curve, as well as to obtain a stress-independent equilibrium Burgers vector and to avoid artificial dissipation during elastic deformation. The Ginzburg-Landau equations are obtained as the linear kinetic relations between the rate of change of the order parameters and the conjugate thermodynamic driving forces. A crystalline energy coefficient for dislocations is defined as a periodic step-wise function of the coordinate along the normal to the slip plane, which provides an energy barrier normal to the slip plane and determines the desired, mesh-independent height of the dislocation bands for any slip system orientation. Gradient energy contains an additional term, which excludes the localization of a dislocation within a height smaller than the prescribed height, but it does not produce artificial interface energy. An additional energy term is introduced that penalizes the interaction of different dislocations at the same point. Non-periodic boundary conditions for dislocations are introduced which include the change of the surface energy due to the exit of dislocations from the crystal. Ob-

\footnotetext{
* Corresponding author. Email address: vlevitas@iastate.edu, Tel. (515)-294-9691 (V. I. Levitas)
} 
tained kinematics, thermodynamics, and kinetics of dislocations at large strains are simplified for small strains and rotations, as well.

Keywords: Phase field approach; Dislocations; Large strains; Nanoscale

\section{Nomenclature}

$\eta \quad$ order parameter for dislocations

$\Omega_{0} \quad$ reference configuration

$\Omega \quad$ actual configuration

$\Omega_{p} \quad$ configuration after elastic unloading

$\boldsymbol{r}_{0} \quad$ position vector in $\Omega_{0}$

$\boldsymbol{r} \quad$ position vector in $\Omega$

$\boldsymbol{u} \quad$ displacement vector

$t \quad$ time

$\boldsymbol{F} \quad$ deformation gradient

$\boldsymbol{F}_{e} \quad$ elastic part of deformation gradient

$\boldsymbol{F}_{p} \quad$ plastic part of deformation gradient

$\boldsymbol{l}_{p} \quad$ plastic velocity gradient

$\varepsilon \quad$ strain tensor

$\boldsymbol{\varepsilon}_{e} \quad$ elastic strain tensor

$\varepsilon_{p} \quad$ plastic strain tensor

$\boldsymbol{\omega} \quad$ skew-symmetric small rotation tensor

$\boldsymbol{\omega}_{e} \quad$ skew-symmetric elastic rotation tensor

$\boldsymbol{\omega}_{p} \quad$ skew-symmetric plastic rotation tensor

$\boldsymbol{n}_{0}^{\alpha} \quad$ unit normal to the slip plane $\alpha$ in $\Omega_{0}$

$\boldsymbol{n}^{\alpha} \quad$ unit normal to the slip plane $\alpha$ in $\Omega$

$\boldsymbol{b}_{0}^{\alpha} \quad$ Burgers vector for the $\alpha^{t h}$ slip system in $\Omega_{0}$

$\boldsymbol{b}^{\alpha} \quad$ Burgers vector for the $\alpha^{t h}$ slip system in $\Omega$

$\boldsymbol{m}_{0}^{\alpha} \quad$ unit vector in the direction of $\boldsymbol{b}_{0}^{\alpha}$

$\boldsymbol{m}^{\alpha} \quad$ unit vector in the direction of $\boldsymbol{b}^{\alpha}$

$\tau \quad$ resolved shear stress for a dislocation

$\gamma \quad$ plastic shear for a single dislocation

$H \quad$ height of a dislocation band

$\boldsymbol{t}_{0} \quad$ traction vector in $\Omega_{0}$
$\boldsymbol{P}$ first Piola-Kirchhoff stress tensor

$\boldsymbol{\sigma} \quad$ Cauchy stress tensor

$f \quad$ body force per unit mass

$\bar{r} \quad$ heat supply per unit mass

$\boldsymbol{v} \quad$ material velocity

$\tilde{S}_{i} \quad$ local entropy production rate

$S_{i} \quad$ total entropy production rate

$s \quad$ specific entropy

$\rho_{0} \quad$ mass density in $\Omega_{0}$

$\rho \quad$ mass density in $\Omega$

$\boldsymbol{\Theta}_{\alpha} \quad$ generalized force conjugate to $\dot{\eta}_{\alpha}$

$U \quad$ internal energy per unit mass

$D \quad$ dissipation rate per unit mass

$X \quad$ dissipative force conjugate to $\dot{\eta}$

$L \quad$ kinetics coefficient

$\beta \quad$ gradient energy coefficient

$\psi \quad$ Helmholtz free energy per unit mass

$\psi^{e} \quad$ elastic energy per unit mass

$\psi^{c} \quad$ crystalline (Peierls) energy per unit mass

$\psi^{\text {int }}$ the energy of interaction of dislocation cores per unit mass

$\psi^{\nabla} \quad$ gradient energy per unit mass

$\boldsymbol{C}^{k} \quad$ elastic moduli tensor of the $k^{\text {th }}$ rank

$\boldsymbol{R}$ orthogonal lattice rotation tensor

$\boldsymbol{U}_{e} \quad$ symmetric elastic right stretch tensor

$V_{e} \quad$ symmetric elastic left stretch tensor

$\boldsymbol{E}_{e} \quad$ Lagrangian elastic strain tensor

$A_{\alpha} \quad$ coefficient characterizing the magnitude of the multi-well crystalline energy

$\bar{A}_{\alpha k} \quad$ coefficient characterizing the magnitude of the energy of interaction of dislocation cores

$M \quad$ ratio of the coefficients for the gradient energy normal to and along the slip plane 


$\begin{array}{llll}\theta & \text { temperature } & \nabla & \text { gradient operator in } \Omega \\ \gamma_{s} & \text { surface energy } & \nabla_{0} & \text { gradient operator in } \Omega_{0} \\ q & \text { Multiplier for the crystalline energy barrier } & \nabla^{2} & \text { Laplacian } \\ J & \text { Jacobian determinant } & \delta i j & \text { Kronecker delta } \\ V_{0} & \text { volume in } \Omega_{0} & \otimes & \text { dyadic product } \\ V & \text { volume in } \Omega & \text { Subscripts and Superscripts } \\ S_{0} & \text { surface in } \Omega_{0} & 0 & \text { undeformed configuration } \\ S & \text { surface in } \Omega & a & \text { skew-symmetric part of a second rank tensor } \\ \boldsymbol{k}_{0} & \text { normal to } S_{0} & e & \text { elastic } \\ \boldsymbol{k} & \text { normal to } S & p & \text { plastic } \\ \boldsymbol{I} & \text { unit tensor } & s & \text { symmetric part of a second rank tensor } \\ \forall & \text { for all } & T & \text { transpose }\end{array}$

\section{Introduction}

The continuum theory of dislocations has been widely used for decades to study dislocation nucleation and evolution as well as plasticity, as seen in recent papers by Cui et al. (2014); Engels et al. (2012); Fan et al. (2011); Huang et al. (2012); Li et al. (2014); Liu et al. (2011); Öztop et al. (2013); Xiong et al. (2014); Yanilkin et al. (2014). A phase field approach (PFA) to dislocations is broadly used for modeling plastic deformation at the nanoscale (Wang et al. (2001a,b,c); Hu and Chen (2001, 2002); Jin and Khachaturyan (2001); Wang et al. (2003); Kundin et al. (2011); Koslowski et al. (2002); Koslowski and Ortiz (2004); Rodney et al. (2003); Shen and Wang (2004); Hu et al. (2004); Wang and Li (2010); Hunter et al. (2010, 2011); Lei and Koslowski (2011); Koslowski (2007); Vorontsov et al. (2004)). The main idea follows from the PFA to martensitic phase transformations (Jin et al. (2001); Chen (2002); Wang and Khachaturyan (1997); Artemev et al. (2001); Levitas and Lee (2007)), in which the deformation of the crystal lattice during stress- or temperature-induced phase transformations and the evolution of martensitic microstructure is determined by the evolution of the order parameters. The evolution of phase interfaces is described in a continuous way by a solution of the Ginzburg-Landau equation without extra efforts to track the interfaces in simulations. The similarity between phase transformations and dislocations is based on Nabarro's idea (Nabarro (1951)) that each dislocation can be described as a thin coherent plate-like inclusion 
with transformation (misfit) strain corresponding to simple shear. For phase transformations, a jump in all properties across a sharp interface is substituted in the PFA by a continuous change of all properties across a finite width interface. Similarly, in the Peierls (1940) and Nabarro (1947) model for dislocations, a jump in displacements by the Burgers vector $\boldsymbol{b}$ is substituted in the PFA by a continuous variation of displacements across the finite width dislocation core. This idea was generalized for an arbitrary three-dimensional system of dislocations in Wang et al. (2001a,b,c); Hu and Chen (2001, 2002); Jin and Khachaturyan (2001); Wang et al. (2003); Koslowski et al. (2002); Koslowski and Ortiz (2004); Rodney et al. (2003); Shen and Wang (2004), utilizing the similarity with phase transformations. The time-dependent GinzburgLandau equation was used to describe the temporal evolution of the order parameter fields, i.e. the dislocation motion, and the elastic problem was solved using the KhachaturyanShatalov microelasticity theory (Wang et al. (2001a,b,c); Hu and Chen (2001, 2002); Jin and Khachaturyan (2001); Wang et al. (2003); Rodney et al. (2003); Shen and Wang (2004)). There is no need to use special numerical approaches to explicitly track dislocation lines. The PFA developed by Koslowski et al. (Koslowski et al. (2002); Koslowski and Ortiz (2004)) is based on an incremental variational framework and characterizes the dislocation evolution through a set of minimization problems. The developed framework was applied to the solutions of a number of material problems, including dislocation evolution in an elastically anisotropic single crystal (Wang et al. (2001a)), polycrystal (Jin and Khachaturyan (2001)), and near a free surface and in heteroepitaxial thin films (Wang et al. (2003)); solute segregation and nucleation of coherent particles around two-dimensional static edge dislocations (Hu and Chen (2001, 2002)). The state of the art of the PFA to dislocations is presented in the recent reviews (Wang and Li (2010); Kundin et al. (2011)). Despite significant progress, there are a number of basic outstanding problems to be resolved.

(I) It was demonstrated in Levitas et al. (2003) that when the Burgers vector is linearly dependent on the order parameters $\eta$ (like in Wang et al. (2001a,b,c); Hu and Chen (2001, 2002); Jin and Khachaturyan (2001); Wang et al. (2003); Kundin et al. (2011); Koslowski et al. (2002); Koslowski and Ortiz (2004); Rodney et al. (2003); Shen and Wang (2004); Wang and Li (2010); Hunter et al. (2010, 2011); Lei and Koslowski (2011); Koslowski (2007); Vorontsov et al. (2004)), the thermodynamically equilibrium value of $\eta$, and consequently the equilibrium 
Burgers vector, depends on the stress tensor $\boldsymbol{\sigma}$. Such a dependence is in conflict with the generally accepted constant (i.e., stress-independent) Burgers vector in the theory of dislocations (Hirth and Lothe (1992)). In addition, stress oscillation in the elastic regime should not be accompanied by energy dissipation in the dislocation (Hirth and Lothe (1992)) and macroscopic plasticity (Levitas (1996)) theories. However, since the order parameter in Wang et al. (2001a,b,c); Hu and Chen (2001, 2002); Jin and Khachaturyan (2001); Wang et al. (2003); Kundin et al. (2011); Koslowski et al. (2002); Koslowski and Ortiz (2004); Rodney et al. (2003); Shen and Wang (2004); Wang and Li (2010); Hunter et al. (2010, 2011); Lei and Koslowski (2011); Koslowski (2007); Vorontsov et al. (2004) varies with stresses in any stress range, and its variation is accompanied by dissipation, this condition is not satisfied. Such an artificial dissipation during elastic deformation is contradictory in principle; however, it may not be critical when loading is monotonous. This problem is very similar to that found in Levitas and Preston (2002a,b) for martensitic phase transformations; namely, that thermodynamic equilibrium transformation strain depends on stresses and temperature which is not the case in the crystallographic theory and sharp interface approach.

(II) While general thermodynamics framework and potentials which satisfy a number of important conditions have been developed for martensitic phase transformations at small (Levitas and Preston (2002a,b); Levitas et al. (2003)) and large (Levitas (2013b)) strains, similar efforts do not exist for dislocations.

(III) All of the previous studies are based on a geometrically linear formulation, i.e., strains and rotations are smaller than 0.1 and are neglected in comparison with 1 . This creates several issues. First, plastic shear for a single dislocation $\gamma=|\boldsymbol{b}| / H$ can be much larger than 0.1 , where $H$ is the dislocation height. For example, when dislocation height $H$ is equal to the interplanar distance $d, \gamma=0.7$ in Zhou et al. (2007) and Lee et al. (2011), and 0.87 in Wang et al. (2001). Second, for $n$ dislocations, plastic shear is equal to $n \gamma$, which is huge for multiple dislocations, even for small plastic shear for single dislocations $\gamma<0.1$. However, in the most larger-scale simulations (Wang et al. (2001b); Jin and Khachaturyan (2001); Wang et al. (2003)), H is chosen to be $\sim 100 d$ and shear strain is spread over 100 interplanar distances and, consequently, reduced by a factor of $H / d$. This does not allow one to resolve the dislocation core and introduces inaccuracy in short-range interactions of dislocations and dislocations with other defects, 
but does not affect stresses far from the dislocations. However, even for such simulations, shear strain $\gamma \sim n d / H$ is finite for $n>0.1 H / d$. Third, not only the plastic shear but also the elastic strains could be finite, because stresses for nucleation of a dislocation are of the order of the theoretical shear strength. Also, large elastic strains and stresses are concentrated at the tip of the dislocation pile-up and are proportional to the number of dislocations $n$, which can be of the order of 10 to 100. Finite elastic strains have to be taken into account when resolving the dislocation core structure and when examining the short-range interaction of dislocations with different defects and phase interfaces, as well as dislocation reactions (Shen and Wang (2004); Hu et al. (2004); Wang and Li (2010)). Fourth, for large strains the spectral methods for the problem solution combined with the Khachaturyan-Shatalov microelasticity theory, developed in Shen and Wang (2004); Wang et al. (2001b); Jin and Khachaturyan (2001); Wang et al. (2003); Hu et al. (2004); Koslowski et al. (2002); Koslowski and Ortiz (2004); Wang and Li (2010), are not applicable because they are based on physically and geometrically linear formulations.

(IV) While the height of a dislocation $H$ is an important parameter in the PFA for dislocations, it is not defined in the theory. Instead, it is assumed in Shen and Wang (2004); Jin and Khachaturyan (2001); Wang et al. (2001b, 2003); Hu et al. (2004) to be equal to the mesh size. This means that there is no objective theory and solutions based on this theory are mesh dependent. As will be seen in Javanbakht and Levitas (2014), mesh dependence is quite drastic and solutions are different not only for different sizes of mesh but also for a structured and slightly unstructured mesh of the same size. The main problem is not related to the numerical approach but in the theoretical deficiency. For phase transformation the contribution to the energy related to the gradient of the order parameter introduces a characteristic size (i.e., interface width) and penalizes interface energy. For a dislocation loop the dislocation core energy is localized along the dislocation line. After the dislocation passes through a given point, the crystal lattice is perfect and does not possess any extra energy. That is why gradient energy does not have a contribution from the gradient of the order parameters along the normal $\boldsymbol{n}$ to the slip plane $\nabla^{n} \eta=\boldsymbol{n} \cdot \nabla \eta$; the gradient of the order parameters along the slip plane, $\nabla^{m} \eta=\boldsymbol{m} \cdot \nabla \eta$, contributes only, where $\boldsymbol{m}=\boldsymbol{b} /|\boldsymbol{b}|$ (Fig. 1). The generic expression for the gradient energy is $\psi^{\nabla}=0.5 \beta\left(\nabla^{m} \eta\right)^{2}$ with the coefficient $\beta$. Characteristic length along the 
slip plane, which is the width of the dislocation core $\delta$, is defined by $\delta \sim \sqrt{\beta / A}$, where $A$ is the magnitude of a multi-well barrier in the crystalline energy. If we assume a similar expression for the gradient energy related to $\nabla^{n} \eta$, i.e., $\psi_{n}^{\nabla}=0.5 \beta_{n}\left(\nabla^{n} \eta\right)^{2}$, then the corresponding characteristic length, which is the height of the dislocation band, $H \sim \sqrt{\beta_{n} / A}$. Since in all theories $\nabla^{n} \eta$ does not contribute to the energy, i.e., $\beta_{n}=0$, then $H=0$. Thus, the problem is ill-posed, similar to the problems for shear band localization in classical plasticity (Abu Al-Rub and Voyiadjis (2005); Zbib and Aifantis (1992)).

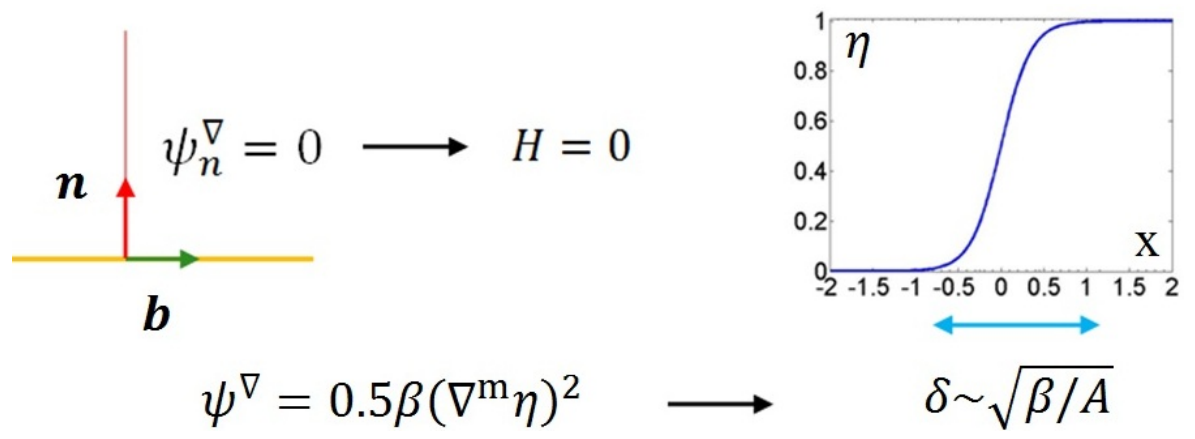

Figure 1: Gradient energy for PFA to dislocations and corresponding characteristic length. Gradient energy contains only the terms along the slip plane, $\psi^{\nabla}=0.5 \beta\left(\nabla^{m} \eta\right)^{2}$, which results in the characteristic length (width of the dislocation core) $\delta \sim \sqrt{\beta / A}$. Due to lack of the gradient term normal to the slip plane, the corresponding characteristic length $H=0$.

In the present work, the phase field approach to dislocations at the nanoscale is conceptually advanced and a thermodynamically consistent theory for large strain is developed. It resolves the above mentioned contradictions and satisfies conditions that are similar to those for phase transformations (Levitas and Preston (2002a,b); Levitas et al. (2003); Levitas (2013b)). This paper is organized as follows. Finite strain kinematics based on multiplicative decomposition of the deformation gradient into elastic and plastic contributions is formulated in Section 2.1. In contrast to all previous phase field formulations for phase transformations and dislocations, in which inelastic strain is expressed as a function of the order parameters, here we formulate an equation for the rate of the plastic deformation gradient versus rate of the order parameters. The application of the first and second laws of thermodynamics for materials for which Helmholtz energy depends on the order parameters and their gradients in Section 2.2 results, in addition to the elasticity rule and expression for the entropy, in the explicit expression for the driving forces for change in order parameters. Requirements for the thermodynamic functions are formulated in Section 3. Two polynomials for plastic transformation strain that satisfy the 
formulated requirements are suggested. The general structure and specific expression for the Helmholtz energy are presented in Section 4. In particular, in order to introduce the height $H$ of any dislocation bands of any orientation, the magnitude of the crystalline energy is chosen to be a periodic step-wise function of the coordinate along the normal to the slip plane. The desired equilibrium stress-order parameter curve is obtained. Gradient energy contains a regularized term related to $\nabla^{n} \eta=\boldsymbol{n} \cdot \boldsymbol{\nabla} \eta$, which excludes the localization of a dislocation within a height smaller than $H$ but does not produce artificial interface energy. An additional energy term is introduced that penalizes the interaction of different dislocations at the same point. In Section 5 the Ginzburg-Landau equations are obtained as the linear kinetic relations between the rate of change of the order parameters and the conjugate thermodynamic driving forces. Boundary conditions for the order parameters in Section 6 take into account the change in surface energy when dislocations exit the crystal. In Section 7 the drawbacks of the previous PFA to dislocations are demonstrated.

Some preliminary results (without derivation and justification) for a simplified case have been presented as a one page summary in the short letter by Levitas and Javanbakht (2012). In Levitas and Javanbakht (2012); Javanbakht and Levitas (2014)), the developed theory is applied to the numerical modeling of several important problems on dislocation nucleation and evolution along single and multiple slip systems as well as dislocations interactions. In Levitas and Javanbakht (2013, 2014), simplified theory by Levitas and Javanbakht (2012) is included into more general PFA to interaction between dislocations and phase transformations and number of problems has been solved using finite element method.

We designate vectors and tensors with boldface symbols and designate contractions of tensors $\boldsymbol{A}=\left\{A_{i j}\right\}$ and $\boldsymbol{B}=\left\{B_{j i}\right\}$ over one and two indices as $\boldsymbol{A} \cdot \boldsymbol{B}=\left\{A_{i j} B_{j k}\right\}$ and $\boldsymbol{A}: \boldsymbol{B}=A_{i j} B_{j i}$; summation is assumed over the repeated indices. In some cases, when it is desirable to show the limits of summations, the sign $\sum$ will be used.

\section{Thermomechanical framework for phase field approach to dis- locations}

\subsection{Kinematics}


Let $\boldsymbol{r}=\boldsymbol{r}\left(\boldsymbol{r}_{0}, t\right)$ be the location of a material point $\boldsymbol{r}_{0}$ of a body at time $t, \boldsymbol{r}\left(\boldsymbol{r}_{0}, 0\right)=\boldsymbol{r}_{0}$, and $\boldsymbol{u}$ be the displacement. The points $\boldsymbol{r}_{0}$ form the reference (undeformed) configuration $\Omega_{0}$ while the points $\boldsymbol{r}$ form the actual (deformed) configuration $\Omega$, i.e., under actual stress and including both elastic and plastic deformations. The multiplicative decomposition of the total deformation gradient, $\boldsymbol{F}:=\partial \boldsymbol{r} / \partial \boldsymbol{r}_{0}=\nabla_{0} \boldsymbol{r}$,

$$
\boldsymbol{F}=\frac{\partial \boldsymbol{r}}{\partial \boldsymbol{p}} \cdot \frac{\partial \boldsymbol{p}}{\partial \boldsymbol{r}_{0}}=\boldsymbol{F}_{e} \cdot \boldsymbol{F}_{p} ; \quad \boldsymbol{F}_{e}:=\frac{\partial \boldsymbol{r}}{\partial \boldsymbol{p}}=\boldsymbol{R} \cdot \boldsymbol{U}_{e}:=\boldsymbol{V}_{e} \cdot \boldsymbol{R} ; \quad \boldsymbol{F}_{p}:=\frac{\partial \boldsymbol{p}}{\partial \boldsymbol{r}_{0}}
$$

into elastic $\boldsymbol{F}_{e}$ and plastic $\boldsymbol{F}_{p}$ parts is used. Here $\boldsymbol{R}$ is the proper orthogonal lattice rotation tensor $\left(\boldsymbol{R}^{T}=\boldsymbol{R}^{-1}\right.$, det $\left.\boldsymbol{R}=1\right) ; \boldsymbol{U}_{e}$ and $\boldsymbol{V}_{e}$ are the symmetric elastic right and left stretch tensors, characterizing deformation of the crystal lattice. Eq.(1) is generally accepted in crystal plasticity (Asaro (1983); Havner (1992); Lubarda (2002)). After a local reduction of the stress tensor in the local vicinity of any point to zero and the disappearance of elastic strain and rotation (i.e., $\boldsymbol{F}_{e}=\boldsymbol{I}$ ), an unloaded configuration characterized by the local position vector $\boldsymbol{p}=\boldsymbol{p}\left(\boldsymbol{r}_{0}, t\right)$ and plastic deformation gradient $\boldsymbol{F}_{p}$, is designated as $\Omega_{p}$. The important point in the unambiguous separation of $\boldsymbol{F}_{e}$ and $\boldsymbol{F}_{p}$ is that $\boldsymbol{F}_{p}$ does not change an orientation of the crystal lattice and represents a combination of lattice-invariant shears. Jacobians, which describe ratios of volumes $V$ and mass densities $\rho$ in different configurations, are defined as

$$
J:=\frac{d V}{d V_{0}}=\frac{\rho_{0}}{\rho}=\operatorname{det} \boldsymbol{F} ; \quad J_{e}:=\frac{d V}{d V_{p}}=\frac{\rho_{p}}{\rho}=\operatorname{det} \boldsymbol{F}_{e} .
$$

Since the plastic deformation is incompressible, $\rho_{0}=\rho_{p}, J_{p}:=d V_{p} / d V_{0}=\rho_{0} / \rho_{p}=1$ and $J=J_{e}$. For small strain and rotation approximation,

$$
F \simeq I+\varepsilon+\omega ; \quad F_{e} \simeq I+\varepsilon_{e}+\omega_{e} ; \quad F_{p} \simeq I+\varepsilon_{p}+\omega_{p}
$$

where $\varepsilon$ is the symmetric small strain tensor and its elastic and plastic components, and $\boldsymbol{\omega}$ is the skew-symmetric small rotation tensor and its elastic and plastic components. Then, neglecting the product of two small tensors, we obtain

$$
\boldsymbol{F}=\left(\boldsymbol{I}+\varepsilon_{e}+\boldsymbol{\omega}_{e}\right) \cdot\left(\boldsymbol{I}+\varepsilon_{p}+\boldsymbol{\omega}_{p}\right) \simeq \boldsymbol{I}+\varepsilon_{e}+\boldsymbol{\omega}_{e}+\varepsilon_{p}+\boldsymbol{\omega}_{p}=\boldsymbol{F}_{e}+\boldsymbol{F}_{p}-\boldsymbol{I}
$$

and a comparison with Eq.(3) for $\boldsymbol{F}$ implies

$$
\varepsilon=\varepsilon_{e}+\varepsilon_{p} ; \quad \omega=\omega_{e}+\omega_{p}
$$

Thus, the multiplicative decomposition of the deformation gradient into elastic and plastic parts at large strains reduces to an additive decomposition of strain and rotations for a geometrically 
linear case. For a single slip $\alpha^{t h}$ with the unit normal $\boldsymbol{n}_{0}^{\alpha}$ with the Burgers vector $\boldsymbol{b}_{0}^{\alpha}, \boldsymbol{F}_{p \alpha}$ represents a simple shear

$$
\boldsymbol{F}_{p \alpha}=\boldsymbol{I}+\frac{1}{H^{\alpha}} \boldsymbol{b}_{0}^{\alpha} \otimes \boldsymbol{n}_{0}^{\alpha} \Phi\left(\eta_{\alpha}\right)=\boldsymbol{I}+\gamma_{\alpha} \boldsymbol{m}_{0}^{\alpha} \otimes \boldsymbol{n}_{0}^{\alpha} \Phi\left(\eta_{\alpha}\right) .
$$

where $\gamma_{\alpha}=\left|\boldsymbol{b}_{0}^{\alpha}\right| / H^{\alpha}$ is the plastic shear strain in a dislocation band of the height $H^{\alpha}$ per single dislocation, $\boldsymbol{m}_{0}^{\alpha}$ is the unit vector in the direction of $\boldsymbol{b}_{0}^{\alpha}, \eta_{\alpha}$ is the order parameter for a dislocation in the $\alpha^{t h}$ slip system, which varies between $n-1$ and $n$ when $n-1$ complete dislocations exist and the $n^{\text {th }}$ dislocation appears; $\Phi$ is a function to be found, which satisfies the condition $\Phi(n)=n$ and some other conditions are formulated below. There is no summation in Eq.(6). All parameters $\left(\boldsymbol{n}_{0}^{\alpha}, \boldsymbol{b}_{0}^{\alpha}, H^{\alpha}, \ldots\right)$ are determined in the undeformed configuration. Thus, the order parameter is unambiguously connected to the magnitude of the Burgers vector $\boldsymbol{b}_{0}^{\alpha} \Phi\left(\eta_{\alpha}\right)$ or plastic shear $\gamma_{\alpha} \Phi\left(\eta_{\alpha}\right)$ in the transitional state between $n-1$ and $n$ dislocations: when $\eta_{\alpha}$ varies between $n-1$ and $n$; the Burgers vector and plastic shear vary between their values for $n-1$ and $n$ dislocations. We define

$$
\begin{gathered}
\dot{\boldsymbol{F}}_{p \alpha}=\frac{1}{H^{\alpha}} \boldsymbol{b}_{0}^{\alpha} \otimes \boldsymbol{n}_{0}^{\alpha} \dot{\Phi}\left(\eta_{\alpha}\right) \\
\boldsymbol{F}_{p \alpha}^{-1}=\boldsymbol{I}-\frac{1}{H^{\alpha}} \boldsymbol{b}_{0}^{\alpha} \otimes \boldsymbol{n}_{0}^{\alpha} \Phi\left(\eta_{\alpha}\right) .
\end{gathered}
$$

which can be checked by proving that $\boldsymbol{F}_{p \alpha} \cdot \boldsymbol{F}_{p \alpha}^{-1}=\boldsymbol{I}$, since vectors $\boldsymbol{n}^{\alpha}$ and $\boldsymbol{b}_{0}^{\alpha}$ are mutually orthogonal $\left(\boldsymbol{b}_{0}^{\alpha} \cdot \boldsymbol{n}_{0}^{\alpha}=0\right)$, and plastic velocity gradient

$$
\boldsymbol{l}_{p}^{\alpha}:=\dot{\boldsymbol{F}}_{p \alpha} \cdot \boldsymbol{F}_{p \alpha}^{-1}=\frac{1}{H^{\alpha}} \boldsymbol{b}_{0}^{\alpha} \otimes \boldsymbol{n}_{0}^{\alpha} \dot{\Phi}\left(\eta_{\alpha}\right)=\dot{\boldsymbol{F}}_{p \alpha}
$$

For finite strains, phenomenological crystal plasticity utilizes the additivity of plastic velocity gradients (Asaro (1983); Havner (1992); Lubarda (2002)). Since we have to be consistent with phenomenological crystal plasticity, we have to accept similar decomposition as well:

$$
\boldsymbol{l}_{p}:=\dot{\boldsymbol{F}}_{p} \cdot \boldsymbol{F}_{p}^{-1}:=\sum_{\alpha=1}^{m} \boldsymbol{l}_{p}^{\alpha}=\sum_{\alpha=1}^{m} \frac{1}{H^{\alpha}} \boldsymbol{b}_{0}^{\alpha} \otimes \boldsymbol{n}_{0}^{\alpha} \dot{\Phi}\left(\eta_{\alpha}\right) .
$$

We explicitly assumed in Eq.(10) that the function $\Phi$ is the same for each slip system and does not contain any interaction (i.e., dependence on $\eta_{i}$ for different slip system) between different slip systems. This is acceptable for the current paper focused on complete dislocations, but should be generalized when partial dislocations and dislocation reactions are included (Wang and Shen (2003); Hunter et al. (2011)). Eq.(10) guarantees plastic incompressibility 
for any values of the order parameters. Indeed, the rate of the volumetric plastic strain is $\dot{J}_{p}=J_{p} \dot{\boldsymbol{F}}_{p \alpha}: \boldsymbol{F}_{p \alpha}^{-1}=J_{p} \boldsymbol{I}: \boldsymbol{l}_{p}^{\alpha}=0$ because $\boldsymbol{b}_{0}^{\alpha} \cdot \boldsymbol{n}_{0}^{\alpha}=0$.

Thus, in contrast to the PFA for martensitic phase transformations and twinning at large strains (Levitas and Preston (2005); Levitas et al. (2009); Clayton and Knap (2011)), in which the finite expression for the transformation strain in terms of the order parameters, $\boldsymbol{F}_{p}\left(\eta_{\alpha}\right)$, was accepted, here we formulate a differential Eq.(10) for $\boldsymbol{F}_{p}$, which can be expressed as

$$
\dot{\boldsymbol{F}}_{p}:=\boldsymbol{l}_{p} \cdot \boldsymbol{F}_{p}=\left[\sum_{\alpha=1}^{m} \frac{1}{H^{\alpha}} \boldsymbol{b}_{0}^{\alpha} \otimes \boldsymbol{n}_{0}^{\alpha} \dot{\Phi}\left(\eta_{\alpha}\right)\right] \cdot \boldsymbol{F}_{p} .
$$

Thus, $\boldsymbol{F}_{p}$ as a solution of Eq.(11) does not only depend on all the order parameters but on the entire history of their variation. This history-dependence cannot be described if we assume relationship for the final plastic deformation gradient $\boldsymbol{F}_{p}\left(\eta_{\alpha}\right)$ rather than the differential Eq.(10). If, e.g., plastic strain occurs along some single slip system 1 producing $\boldsymbol{F}_{p 1}$, then along some single slip system 2 at fixed $\boldsymbol{F}_{p 1}$ producing $\boldsymbol{F}_{p 2}$ on the top of $\boldsymbol{F}_{p 1}$, and so on, then Eq.(11) can be integrated as

$$
\boldsymbol{F}_{p}=\boldsymbol{F}_{p m} \cdot \ldots \cdot \boldsymbol{F}_{p 2} \cdot \boldsymbol{F}_{p 1} ; \quad \boldsymbol{F}_{p \alpha}=\boldsymbol{I}+\frac{1}{H^{\alpha}} \boldsymbol{b}_{0}^{\alpha} \otimes \boldsymbol{n}_{0}^{\alpha} \Phi\left(\eta_{\alpha}\right)
$$

Indeed, if $\boldsymbol{F}_{p m}$ varies only while all previous slips are fixed, then

$$
\begin{aligned}
& \dot{\boldsymbol{F}}_{p}=\dot{\boldsymbol{F}}_{p m} \cdot \ldots \cdot \boldsymbol{F}_{p 2} \cdot \boldsymbol{F}_{p 1} ; \quad \boldsymbol{F}_{p}^{-1}=\boldsymbol{F}_{p 1}^{-1} \cdot \boldsymbol{F}_{p 2}^{-1} \cdot \ldots \cdot \boldsymbol{F}_{p m}^{-1} \\
& \boldsymbol{l}_{p}:=\dot{\boldsymbol{F}}_{p} \cdot \boldsymbol{F}_{p}^{-1}=\dot{\boldsymbol{F}}_{p m} \cdot \boldsymbol{F}_{p m}^{-1}=\dot{\boldsymbol{F}}_{p m}=\frac{1}{H^{m}} \boldsymbol{b}_{0}^{m} \otimes \boldsymbol{n}_{0}^{m} \dot{\Phi}\left(\eta_{m}\right) .
\end{aligned}
$$

where Eq.(9) was utilized for a simple shear $\boldsymbol{F}_{p m}$. The last Eq.(13) can be integrated

$$
\boldsymbol{F}_{p m}=\boldsymbol{I}+\frac{1}{H^{m}} \boldsymbol{b}_{0}^{m} \otimes \boldsymbol{n}_{0}^{m} \Phi\left(\eta_{m}\right) .
$$

and it is independent of previous $\boldsymbol{F}_{p \alpha}$. Thus, it can be multiplicatively superposed on the deformation gradient due to previous shears. Formally, Eq.(12) can be applied when each shear occurs along the same slip system with increasing number of dislocations. In this case, it transforms to an additive rule, e.g.,

$$
\begin{aligned}
& \boldsymbol{F}_{p}=\boldsymbol{F}_{p 2} \cdot \boldsymbol{F}_{p 1}=\left(\boldsymbol{I}+\frac{1}{H^{\alpha}} \boldsymbol{b}_{0}^{\alpha} \otimes \boldsymbol{n}_{0}^{\alpha}\left(\Phi\left(\eta_{\alpha}\right)-\Phi\left(\eta_{\alpha}^{1}\right)\right)\right) \cdot\left(\boldsymbol{I}+\frac{1}{H^{\alpha}} \boldsymbol{b}_{0}^{\alpha} \otimes \boldsymbol{n}_{0}^{\alpha} \Phi\left(\eta_{\alpha}^{1}\right)\right)= \\
& \boldsymbol{I}+\frac{1}{H^{\alpha}} \boldsymbol{b}_{0}^{\alpha} \otimes \boldsymbol{n}_{0}^{\alpha} \Phi\left(\eta_{\alpha}\right) .
\end{aligned}
$$

because $\boldsymbol{n}_{0}^{\alpha} \cdot \boldsymbol{b}_{0}^{\alpha}=0$, i.e., our initial assumption of the additivity of shears along the same slip direction is noncontradictory. 
To summarize, Eq.(10) (or Eq.(11)) will be used as our main kinematic relationship.

For small strains, when slip occurs simultaneously along several slip planes and systems, Eq.(11) reduces to

$$
\dot{\boldsymbol{\varepsilon}}_{p}+\dot{\boldsymbol{\omega}}_{p}=\sum_{\alpha=1}^{m} \frac{1}{H^{\alpha}} \boldsymbol{b}_{0}^{\alpha} \otimes \boldsymbol{n}_{0}^{\alpha} \dot{\Phi}\left(\eta_{\alpha}\right) .
$$

This equation can be integrated explicitly, and we obtain an additive decomposition for plastic strains and rotations

$$
\boldsymbol{\varepsilon}_{p}+\boldsymbol{\omega}_{p}=\sum_{\alpha=1}^{m} \frac{1}{H^{\alpha}} \boldsymbol{b}_{0}^{\alpha} \otimes \boldsymbol{n}_{0}^{\alpha} \Phi\left(\eta_{\alpha}\right),
$$

which is usually accepted for small strain formulation (Wang et al. (2001b)), similar to crystal plasticity. It preserves plastic incompressibility, because volumetric plastic strain

$$
\varepsilon_{v p}:=J_{p}-1:=\left(\varepsilon_{p}+\omega_{p}\right): \boldsymbol{I}=\sum_{\alpha=1}^{m} \frac{1}{H^{\alpha}} \boldsymbol{b}_{0}^{\alpha} \cdot \boldsymbol{n}_{0}^{\alpha} \Phi\left(\eta_{\alpha}\right)=0 .
$$

\subsection{Application of thermodynamic laws}

We will consider an arbitrary volume $V_{0}$ of a material bounded by a surface $S_{0}$ with unit external normal $\boldsymbol{k}_{0}$ in the undeformed configuration. This volume can be cut from any actual body, thus $S_{0}$ is not an external surface and the surface energy will not be included in the thermodynamics laws. The first law of thermodynamics for the volume $V_{0}$ in the reference configuration can be presented in the form of the global (i.e., for the entire body) energy balance equation

$$
\begin{aligned}
& \int_{S_{0}}\left(\boldsymbol{t}_{0} \cdot \boldsymbol{v}-\boldsymbol{h}_{0} \cdot \boldsymbol{k}_{0}\right) d S_{0}+\int_{S_{0}} \Theta_{\alpha}^{0} \dot{\eta}_{\alpha} \cdot \boldsymbol{k}_{0} d S_{0}+\int_{V_{0}} \rho_{0}(\boldsymbol{f} \cdot \boldsymbol{v}+\bar{r}) d V_{0}= \\
& \frac{d}{d t} \int_{V_{0}} \rho_{0}(U+0.5 \boldsymbol{v} \cdot \boldsymbol{v}) d V_{0} .
\end{aligned}
$$

Here $\boldsymbol{t}_{0}$ is the traction vector, $\boldsymbol{v}=\dot{\boldsymbol{r}}$ is the material velocity, $\boldsymbol{h}_{0}$ is the heat flux, $U$ is the specific (per unit mass) internal energy, $\boldsymbol{f}$ is the body force and $\bar{r}$ is the specific volumetric heat supply rate (e.g., due to electric heating), both per unit mass. To balance the terms related to the dependence of the thermodynamic potential on the gradient of the order parameter $\nabla_{0} \eta_{\alpha}$, one has to include extra generalized forces $\Theta_{\alpha}^{0} \cdot \boldsymbol{k}_{0}$ at the surface, of which the tractions are work-conjugate to the rate of change of the order parameters $\dot{\eta}_{\alpha}$. Otherwise, Eq.(19) cannot be applicable for an arbitrary volume. Without this term, Eq.(19) and Eq.(20) 
below can be found in any text book on continuum mechanics, e.g., in Malvern (1977); Lubarda (2002); Lurie (1990). Summation over $\alpha$ is assumed in Eq.(19). Introducing $s, S_{i}$, and $\theta \geq 0$ as the specific entropy, the total entropy production rate, and the temperature, respectively, we will accept the second law of thermodynamics in the combined form of the Clausius-Duhem inequality and the global entropy balance equation for the whole volume $V_{0}$ :

$$
S_{i}:=\frac{d}{d t} \int_{V_{0}} \rho_{0} s d V_{0}-\int_{V_{0}} \rho_{0} \frac{\bar{r}}{\theta} d V_{0}+\int_{S_{0}} \frac{\boldsymbol{h}_{0}}{\theta} \cdot \boldsymbol{k}_{0} d S_{0} \geq 0 .
$$

Using the relationship $\boldsymbol{t}_{0}=\boldsymbol{P} \cdot \boldsymbol{k}_{0}$ between the traction vector and the first Piola-Kirchhoff stress tensor, $\boldsymbol{P}$, and the Gauss theorem, surface integrals can be transformed into integrals over the volume. After some transformations we obtain

$$
\begin{aligned}
& \int_{V_{0}}\left(\boldsymbol{P}: \dot{\boldsymbol{F}}^{T}-\rho_{0} \dot{U}-\nabla_{0} \cdot \boldsymbol{h}_{0}+\rho_{0} \bar{r}+\nabla_{0} \cdot\left(\boldsymbol{\Theta}_{\alpha}^{0} \dot{\eta}_{\alpha}\right)\right) d V_{0}+ \\
& \int_{V_{0}}\left(\nabla_{0} \cdot \boldsymbol{P}+\rho_{0} \boldsymbol{f}-\rho_{0} \dot{\boldsymbol{v}}\right) \cdot \boldsymbol{v} d V_{0}=0 ; \\
& S_{i}:=\int_{V_{0}}\left(\rho_{0} \dot{s}-\rho_{0} \frac{\bar{r}}{\theta}+\nabla_{0} \cdot \frac{\boldsymbol{h}_{0}}{\theta}\right) d V_{0} \geq 0 .
\end{aligned}
$$

The velocity $\boldsymbol{v}$ in Eq.(21) should be substituted with $\boldsymbol{v}-\boldsymbol{v}_{a}$ when the observer moves with respect to a fixed frame of reference at the constant velocity $\boldsymbol{v}_{a}$. Since the energy balance should be indifferent with respect to the change of an observer system, and $\boldsymbol{v}_{a}$ is an arbitrary vector, the term in parenthesis in the second integral in Eq.(21) is identically zero, which leads to $\nabla_{0} \cdot \boldsymbol{P}+\rho_{0} \boldsymbol{f}=\rho_{0} \dot{\boldsymbol{v}}$. Therefore, the extra generalized forces $\boldsymbol{\Theta}_{\alpha}^{0}$ do not affect the local momentum balance equation. Since the equality (21) and the inequality (22) are valid for an arbitrary volume, they have to be valid for each material point:

$$
\begin{gathered}
\boldsymbol{P}: \dot{\boldsymbol{F}}^{T}-\rho_{0} \dot{U}-\nabla_{0} \cdot \boldsymbol{h}_{0}+\rho_{0} \bar{r}+\nabla_{0} \cdot\left(\boldsymbol{\Theta}_{\alpha}^{0} \dot{\eta}_{\alpha}\right)=0 ; \\
\rho_{0} \tilde{S}_{i}:=\rho_{0} \dot{s}-\rho_{0} \frac{\bar{r}}{\theta}+\nabla_{0} \cdot \frac{\boldsymbol{h}_{0}}{\theta}=\rho_{0} \dot{s}-\rho_{0} \frac{\bar{r}}{\theta}+\frac{1}{\theta} \nabla_{0} \cdot \boldsymbol{h}_{0}-\frac{\nabla_{0} \theta}{\theta^{2}} \cdot \boldsymbol{h}_{0} \geq 0,
\end{gathered}
$$

where $\tilde{S}_{i}$ is the local entropy production rate. Let us introduce the local dissipation rate,

$$
\rho_{0} \mathcal{D}:=\rho_{0} \theta \tilde{S}_{i}=\rho_{0} \theta \dot{s}-\rho_{0} \bar{r}+\nabla_{0} \cdot \boldsymbol{h}_{0}-\frac{1}{\theta} \boldsymbol{h}_{0} \cdot \nabla_{0} \theta \geq 0 .
$$

If we resolve Eq.(23) for the term $\nabla_{0} \cdot h_{0}-\rho_{0} \bar{r}$ and substitute it in Eq.(25), we will obtain the 
following dissipation inequality

$$
\rho_{0} \mathcal{D}:=P: \dot{\boldsymbol{F}}^{T}-\rho_{0} \dot{U}+\rho_{0} \theta \dot{s}+\nabla_{0} \cdot\left(\boldsymbol{\Theta}_{\alpha}^{0} \dot{\eta}_{\alpha}\right)-\frac{\boldsymbol{\nabla}_{0} \theta}{\theta} \cdot \boldsymbol{h}_{0} \geq 0 .
$$

It is assumed that the heat conduction and other thermomechanical processes are thermodynamically mutually independent. Thus, we can transform the inequality Eq.(26) into two inequalities: the Fourier's inequality $\frac{\boldsymbol{\nabla}_{0} \theta}{\theta} \cdot \boldsymbol{h}_{0} \geq 0$, and dissipation inequality Eq.(26) without the last term. Introducing the specific Helmholtz free energy per undeformed volume $\psi=U-\theta s$, the final expression for the dissipation inequality will be obtained as

$$
\rho_{0} \mathcal{D}=P: \dot{F}^{T}-\rho_{0} \dot{\psi}-\rho_{0} s \dot{\theta}+\nabla_{0} \cdot\left(\boldsymbol{\Theta}_{\alpha}^{0} \dot{\eta}_{\alpha}\right) \geq 0
$$

Let $\psi=\bar{\psi}\left(\boldsymbol{F}_{e}, \eta_{\alpha}, \nabla_{0} \eta_{\alpha}\right)$-i.e., the free energy is independent of the plastic deformation gradient and the temperature is omitted for compactness. Under superposition of the rigid-body rotation $\boldsymbol{F}^{*}=\boldsymbol{Q} \cdot \boldsymbol{F}$, where $\boldsymbol{Q}$ is the arbitrary proper orthogonal tensor, one has $\boldsymbol{F}_{e}^{*}=\boldsymbol{Q} \cdot \boldsymbol{F}_{e}$ and $\psi=\bar{\psi}\left(\boldsymbol{Q} \cdot \boldsymbol{F}_{e}, \eta_{\alpha}, \boldsymbol{\nabla}_{0} \eta_{\alpha}\right)$. According to the principle of objectivity, energy $\psi$ should be independent of $\boldsymbol{Q}$. As usual (see, e.g., Malvern (1977); Lubarda (2002); Lurie (1990)), utilizing $\boldsymbol{Q}=\boldsymbol{R}^{T}$ and the polar decomposition Eq. (1), one has $\psi=\bar{\psi}\left(\boldsymbol{U}_{e}, \eta_{\alpha}, \boldsymbol{\nabla}_{0} \eta_{\alpha}\right)=\psi\left(\boldsymbol{E}_{e}, \eta_{\alpha}, \boldsymbol{\nabla}_{0} \eta_{\alpha}\right)$, where $\boldsymbol{E}_{e}=0.5\left(\boldsymbol{U}_{e} \cdot \boldsymbol{U}_{e}-\mathbf{I}\right)=0.5\left(\boldsymbol{F}_{e}^{T} \cdot \boldsymbol{F}_{e}-\boldsymbol{I}\right)$ is the Lagrangian elastic strain tensor. Differentiating Eq.(1), we obtain $\dot{\boldsymbol{F}}^{T}=\dot{\boldsymbol{F}}_{p}^{T} \cdot \boldsymbol{F}_{e}^{T}+\boldsymbol{F}_{p}^{T} \cdot \dot{\boldsymbol{F}}_{e}^{T}$. Using

$$
\boldsymbol{\nabla}_{0} \cdot\left(\boldsymbol{\Theta}_{\alpha}^{0} \dot{\eta}_{\alpha}\right)=\left(\boldsymbol{\nabla}_{0} \cdot \boldsymbol{\Theta}_{\alpha}^{0}\right) \dot{\eta}_{\alpha}+\boldsymbol{\Theta}_{\alpha}^{0} \cdot \nabla_{0} \dot{\eta}_{\alpha}
$$

$\nabla_{0} \dot{\eta}_{\alpha}=\overline{\nabla_{0} \eta_{\alpha}}$ and substituting these equations in Eq.(27), one transforms

$$
\begin{aligned}
& \rho_{0} D=\left(\boldsymbol{P} \cdot \boldsymbol{F}_{p}^{T}-\rho_{0} \frac{\partial \psi}{\partial \boldsymbol{F}_{e}}\right) \dot{\boldsymbol{F}}_{e}^{T}+\left(\boldsymbol{F}_{e}^{T} \cdot \boldsymbol{P}: \frac{\partial \boldsymbol{F}_{p}^{T}}{\partial \eta_{\alpha}}+\nabla_{0} \cdot \boldsymbol{\Theta}_{\alpha}^{0}-\rho_{0} \frac{\partial \psi}{\partial \eta_{\alpha}}\right) \dot{\eta}_{\alpha} \\
& \left(\boldsymbol{\Theta}_{\alpha}^{0}-\rho_{0} \frac{\partial \psi}{\partial \boldsymbol{\nabla}_{0} \eta_{\alpha}}\right) \cdot \frac{\cdot}{\nabla_{0} \eta_{\alpha}} \geq 0 .
\end{aligned}
$$

Assuming that the dissipation rate is independent of $\dot{\boldsymbol{F}}_{e}^{T}$ and $\overline{\nabla_{0} \eta_{\alpha}}$, one obtains the constitutive relationship for stresses and definition of $\Theta_{\alpha}^{0}$ :

$$
\boldsymbol{P} \cdot \boldsymbol{F}_{p}^{T}=\rho_{0} \frac{\partial \psi}{\partial \boldsymbol{F}_{e}} ; \quad \boldsymbol{\Theta}_{\alpha}^{0}=\rho_{0} \frac{\partial \psi}{\partial \boldsymbol{\nabla}_{0} \eta_{\alpha}} .
$$

One can assume that the dissipation rate depends on $\overline{\nabla_{0} \eta_{\alpha}}$ and adds a dissipative contribution to the generalized force $\Theta_{\alpha}^{0}$ in Eq.(30). However, then we will not obtain a traditional structure of the Ginzburg-Landau equations, as seen in Section 5. We can also add dissipative stresses in Eq.(30), as it was done for phase transformations in Levitas (2013c, 2014). However, we 
would like to focus on the dislocation glide as the only dissipation mechanism. With Eqs.(30), the dissipation inequality reduces to

$$
D=X_{\alpha} \dot{\eta}_{\alpha} \geq 0 ; \quad \rho_{0} X_{\alpha}:=\boldsymbol{P}^{T} \cdot \boldsymbol{F}_{e}: \frac{\partial \boldsymbol{F}_{p}}{\partial \eta_{\alpha}}+\boldsymbol{\nabla}_{0} \cdot\left(\rho_{0} \frac{\partial \psi}{\partial \boldsymbol{\nabla}_{0} \eta_{\alpha}}\right)-\rho_{0} \frac{\partial \psi}{\partial \eta_{\alpha}},
$$

where $X_{\alpha}$ is the driving force for the change in $\eta_{\alpha}$, i.e., for dislocation evolution. Let us elaborate the plastic work term in Eq.(31) with the help of Eq.(11). Thus, according to Eq.(11)

$$
\frac{\partial \boldsymbol{F}_{p}}{\partial \eta_{\alpha}}=\left[\gamma_{\alpha} \boldsymbol{m}_{0}^{\alpha} \otimes \boldsymbol{n}_{0}^{\alpha} \frac{d \Phi}{d \eta_{\alpha}}\right] \cdot \boldsymbol{F}_{p}
$$

Then

$$
\begin{aligned}
& \boldsymbol{P}^{T} \cdot \boldsymbol{F}_{e}: \frac{\partial \boldsymbol{F}_{p}}{\partial \eta_{\alpha}}=\boldsymbol{P}^{T} \cdot \boldsymbol{F}_{e}:\left[\gamma_{\alpha} \boldsymbol{m}_{0}^{\alpha} \otimes \boldsymbol{n}_{0}^{\alpha} \frac{d \Phi}{d \eta_{\alpha}}\right] \cdot \boldsymbol{F}_{p}= \\
& \boldsymbol{n}_{0}^{\alpha} \cdot \boldsymbol{F}_{p} \cdot \boldsymbol{P}^{T} \cdot \boldsymbol{F}_{e} \cdot \boldsymbol{m}_{0}^{\alpha} \gamma_{\alpha} \frac{d \Phi}{d \eta_{\alpha}}=\tau_{\alpha} \gamma_{\alpha} \frac{d \Phi}{d \eta_{\alpha}} \\
& \tau_{\alpha}:=\boldsymbol{n}_{0}^{\alpha} \cdot \boldsymbol{F}_{p} \cdot \boldsymbol{P}^{T} \cdot \boldsymbol{F}_{e} \cdot \boldsymbol{m}_{0}^{\alpha} .
\end{aligned}
$$

where $\tau_{\alpha}$ is the resolved shear stress for the slip system $\alpha$. With the help of the Cauchy stress $\boldsymbol{\sigma}$ (force per unit deformed area),

$$
\boldsymbol{\sigma}=\frac{1}{J} \boldsymbol{P} \cdot \boldsymbol{F}^{T}=\frac{1}{J} \boldsymbol{P} \cdot \boldsymbol{F}_{p}^{T} \cdot \boldsymbol{F}_{e}^{T}=\rho \frac{\partial \psi}{\partial \boldsymbol{F}_{e}} \cdot \boldsymbol{F}_{e}^{T},
$$

the resolved shear stress can be presented in the form

$$
\tau_{\alpha}=J \boldsymbol{n}_{0}^{\alpha} \cdot \boldsymbol{F}_{e}^{-1} \cdot \boldsymbol{\sigma} \cdot \boldsymbol{F}_{e} \cdot \boldsymbol{m}_{0}^{\alpha}
$$

Thus, Eq.(31) for the driving force for dislocation evolution reduces to

$$
\rho_{0} X_{\alpha}=\tau_{\alpha} \gamma_{\alpha} \frac{\partial \Phi}{\partial \eta_{\alpha}}+\nabla_{0} \cdot\left(\rho_{0} \frac{\partial \psi}{\partial \nabla_{0} \eta_{\alpha}}\right)-\rho_{0} \frac{\partial \psi}{\partial \eta_{\alpha}} .
$$

For small strains and rotations, $\boldsymbol{P} \simeq \boldsymbol{\sigma}, \boldsymbol{F}_{p} \simeq \boldsymbol{F}_{e} \simeq \boldsymbol{I}$, and $\tau_{\alpha}=\boldsymbol{n}^{\alpha} \cdot \boldsymbol{\sigma} \cdot \boldsymbol{m}^{\alpha}$ simplifies to the usual resolved shear stress.

\section{Requirements to the thermodynamic functions}

\subsection{Thermodynamic equilibrium for homogeneous states}

When the order parameters were introduced in Section 2.1, one of the main conditions was that for $n$ complete dislocations passed through the given material point one has $\eta_{\alpha}=$ $n$. Otherwise, the Burgers vector for $n$ dislocations in the slip system $\alpha$ will not be $n \boldsymbol{b}$, as desired. However, it is not sufficient to impose this condition verbally, it should follow from the thermodynamic equilibrium conditions and should be independent of applied stresses; 
otherwise, the Burgers vector will be stress-dependent. Here we determine the limitations on the functions $\Phi\left(\eta_{\alpha}\right)$ and the local part of the free energy $\psi^{l}\left(\boldsymbol{E}_{e}, \eta_{\alpha}\right)$, which are mandatory to satisfy these conditions.

For the thermodynamic equilibrium one has $X_{\alpha}=0$, where for a homogeneous states in Eq.(31) for $X_{\alpha}$ the gradient terms should be omitted. Since dislocations themselves represent heterogeneities (like interfaces for phase transformations), the concept of a homogeneous state with dislocations has to be defined. Thus, a homogeneous state represents homogeneous (perfect) crystal lattice after passing $n_{\beta}$ complete dislocations along each $\beta$ slip system. These dislocations either produce steps at the external surface or are considered to be far from the volume which is under study, so that they do not affect its homogeneity in terms of structure and stresses. While it seems trivial, the application of the thermodynamic equilibrium conditions will lead to nontrivial constraints for thermodynamic and kinematic functions. We formulate the following conditions:

Condition I: the integer values of the order parameter $\eta_{i}=n_{i}$ for any slip system $i$ should satisfy the thermodynamic equilibrium conditions for any slip system $\alpha$

$$
\begin{aligned}
& \rho_{0} X_{\alpha}\left(\eta_{i}=n_{i}\right):=\boldsymbol{P}^{T} \cdot \boldsymbol{F}_{e}: \frac{\partial \boldsymbol{F}_{p}\left(\eta_{i}=n_{i}\right)}{\partial \eta_{\alpha}}-\rho_{0} \frac{\partial \psi^{l}\left(\boldsymbol{E}_{e}, \eta_{i}=n_{i}\right)}{\partial \eta_{\alpha}}=0 ; \\
& \text { for each } \alpha, i=1,2, \ldots, n .
\end{aligned}
$$

for any stress $\boldsymbol{P}$, plastic deformation gradient $\boldsymbol{F}_{p}$, and corresponding elastic deformation gradient $\boldsymbol{F}_{e}$.

Note that when a finite number of complete dislocations passed through the point under study along any slip system, the crystal lattice in the given point does not change. That is why this should not affect thermodynamic equilibrium for dislocations for any other or the same slip systems, which is assumed in Condition I. Since function $\frac{\partial \boldsymbol{F}_{p}}{\partial \eta_{\alpha}}$ is independent of stresses, Condition I can be satisfied when $\frac{\partial \boldsymbol{F}_{p}\left(\eta_{i}=n_{i}\right)}{\partial \eta_{\alpha}}=0$ only. Then the second term in Eq.(39) should also be zero. Also, since according to Eq.(32) $\frac{\partial \boldsymbol{F}_{p}}{\partial \eta_{\alpha}}$ depends on $\eta_{\alpha}$ for the same $\alpha$ and $\omega$ only, Eq.(39) results in two sets of equations:

$$
\begin{aligned}
& \frac{\partial \boldsymbol{F}_{p}\left(\eta_{i}=n_{i}\right)}{\partial \eta_{\alpha}}=\frac{d \Phi\left(\eta_{\alpha}=n_{\alpha}\right)}{d \eta_{\alpha}}=0 ; \quad \frac{\partial \psi^{l}\left(\boldsymbol{E}_{e}, \eta_{i}=n_{i}\right)}{\partial \eta_{\alpha}}=0 ; \\
& \text { for each } \alpha, i=1,2, \ldots, n .
\end{aligned}
$$




\subsection{Instability of thermodynamic equilibrium}

The stability (or instability) of a homogeneous equilibrium state will be considered only. A violation of the stability of the homogeneous equilibrium state will lead to localized plastic shear along single or multiple slip planes by a corresponding Burgers vector, homogeneous within the slip band, i.e., to increase (or decrease) the number of dislocations by one along single or multiple slip systems. Thus instability changes the number of dislocations in any $i$ slip system from $n_{i}$ to $n_{i}+1$ or $n_{i}-1$. We will start with stability under a prescribed first Piola-Kirchhoff stress $P$.

The following thermodynamic definition of shear lattice instability is accepted: the thermodynamic equilibrium with order parameters $\eta_{i}=n_{i}$ is unstable if a spontaneous deviation of any or all order parameters $\Delta \eta_{i}$ is thermodynamically admissible under prescribed boundary conditions, i.e., $D \geq 0$. Thus, thermodynamic instability conditions for $\boldsymbol{P}=$ const are

$$
X_{\alpha}\left(\boldsymbol{P}, \boldsymbol{F}_{e}+\Delta \boldsymbol{F}_{e}, n_{i}+\Delta \eta_{i}\right) \dot{\eta}_{\alpha} \geq 0
$$

where $\Delta \boldsymbol{F}_{e}$ is a spontaneous change in $\boldsymbol{F}_{e}$ due to a change in order parameters under $\boldsymbol{P}=$ const. Using the Taylor expansion of $X_{\alpha}$ around the equilibrium state $\eta_{i}=n_{i}$ and considering equilibrium condition $X_{\alpha}\left(\boldsymbol{P}, \boldsymbol{F}_{e}, \eta_{i}\right)=0$, we obtain from Eq.(41):

$$
\begin{aligned}
& \left.\frac{\partial X_{\alpha}\left(\boldsymbol{P}, \boldsymbol{F}_{e}, \eta_{i}=n_{i}\right)}{\partial \eta_{k}}\right|_{\boldsymbol{P}} \dot{\eta}_{\alpha} \dot{\eta}_{k}= \\
& \left(\frac{\partial X_{\alpha}\left(\boldsymbol{P}, \boldsymbol{F}_{e}, \eta_{i}=n_{i}\right)}{\partial \boldsymbol{F}_{e}^{T}}: \frac{\partial \boldsymbol{F}_{e}}{\partial \eta_{k}}+\left.\frac{\partial X_{\alpha}\left(\boldsymbol{P}, \boldsymbol{F}_{e}, \eta_{i}=n_{i}\right)}{\partial \eta_{i}}\right|_{\boldsymbol{F}_{e}}\right) \dot{\eta}_{\alpha} \dot{\eta}_{k} \geq 0 .
\end{aligned}
$$

where a summation over repeated subscripts is assumed. If the matrix $\left.\frac{\partial X_{\alpha}}{\partial \eta_{k}}\right|_{\boldsymbol{P}}$ is negative definite, condition (42) is not met and the equilibrium is stable. The instability happens when

the matrix $\left.\frac{\partial X_{\alpha}}{\partial \eta_{k}}\right|_{\boldsymbol{P}}$ first ceases to become negative definite. Using the expression for $X_{\alpha}$ from Eq.(31), the $\left.\frac{\partial X_{\alpha}}{\partial \eta_{k}}\right|_{\boldsymbol{P}}$ can be written as

$$
\begin{aligned}
& \left.\frac{\partial X_{\alpha}}{\partial \eta_{k}}\right|_{\boldsymbol{P}}=\frac{1}{\rho_{0}} \boldsymbol{P}^{T} \cdot \frac{\partial \boldsymbol{F}_{e}}{\partial \eta_{k}}: \frac{\partial \boldsymbol{F}_{p}}{\partial \eta_{\alpha}}+\frac{1}{\rho_{0}} \boldsymbol{P}^{T} \cdot \boldsymbol{F}_{e}: \frac{\partial^{2} \boldsymbol{F}_{p}}{\partial \eta_{\alpha}^{2}} \delta_{k \alpha}-\left.\frac{\partial^{2} \psi^{l}}{\partial \eta_{\alpha} \partial \eta_{k}}\right|_{\boldsymbol{F}_{e}}- \\
& \frac{\partial^{2} \psi^{l}}{\partial \eta_{\alpha} \partial \boldsymbol{F}_{e}}:\left.\frac{\partial \boldsymbol{F}_{e}^{T}}{\partial \eta_{k}}\right|_{\boldsymbol{P}} .
\end{aligned}
$$

Now, using the constitutive Eq.(30), the last term of Eq.(43) can be expressed as

$$
\frac{\partial^{2} \psi}{\partial \eta_{\alpha} \partial \boldsymbol{F}_{e}}: \frac{\partial \boldsymbol{F}_{e}^{T}}{\partial \eta_{k}}=\frac{1}{\rho_{0}} \frac{\partial \boldsymbol{P} \cdot \boldsymbol{F}_{p}^{T}}{\partial \eta_{\alpha}}: \frac{\partial \boldsymbol{F}_{e}^{T}}{\partial \eta_{k}}=\frac{1}{\rho_{0}} \boldsymbol{P}^{T} \cdot \frac{\partial \boldsymbol{F}_{e}}{\partial \eta_{k}}: \frac{\partial \boldsymbol{F}_{p}}{\partial \eta_{\alpha}} .
$$


This term and the first term in Eq.(43) eliminate each other and Eq.(43) simplifies to

$$
\left.\frac{\partial X_{\alpha}}{\partial \eta_{k}}\right|_{\boldsymbol{P}}=\frac{1}{\rho_{0}} \boldsymbol{P}^{T} \cdot \boldsymbol{F}_{e}: \frac{\partial^{2} \boldsymbol{F}_{p}}{\partial \eta_{\alpha}^{2}} \delta_{k \alpha}-\left.\frac{\partial^{2} \psi^{l}}{\partial \eta_{\alpha} \partial \eta_{k}}\right|_{\boldsymbol{E}_{e}} \cdot
$$

Substituting Eq.(45) in the instability condition Eq.(42), one simplifies

$$
\left(\frac{1}{\rho_{0}} \boldsymbol{P}^{T} \cdot \boldsymbol{F}_{e}: \frac{\partial^{2} \boldsymbol{F}_{p}\left(\eta_{\alpha}=n_{\alpha}\right)}{\partial \eta_{\alpha}^{2}} \delta_{k \alpha}-\left.\frac{\partial^{2} \psi^{l}\left(\boldsymbol{E}_{e}, \eta_{i}=n_{i}\right)}{\partial \eta_{\alpha} \partial \eta_{k}}\right|_{\boldsymbol{E}_{e}}\right) \dot{\eta}_{\alpha} \dot{\eta}_{k} \geq 0
$$

The first term in Eq.(46) represents the driving force for the lattice instability and the second term characterizes the resistance to the lattice instability. In contrast to martensitic transformations, where the transformation of one martensitic variant into another one is important, the transformation of a complete dislocation in one slip system $\eta_{i}=n_{i}$ into complete dislocation of any other slip system $\alpha$ does not occur and is not of interest for the description. Our interest is to describe the transition from $\eta_{\alpha}=n_{\alpha}$ to $\eta_{\alpha}=n_{\alpha}+1$ or $\eta_{\alpha}=n_{\alpha}-1$ for fixed $\eta_{i}=n_{i}$ for any other slip system, i.e., the appearance or disappearance of one dislocation along the slip system under consideration $\alpha$ for any unchanged number of complete dislocations $\eta_{i}=n_{i}$ for any other slip system. That is why the driving force for instability in Eq.(46) exists for the same slip system $\alpha$ only, which was imposed by the choice of independence of $\boldsymbol{F}_{p}$ of a slip system $\alpha$ on any other $\eta_{k}$. It is reasonable to impose that the variation of $\eta_{\alpha}$ from $n_{\alpha}$ to $n_{\alpha} \pm 1$ occurs at constant $\eta_{i}=n_{i}$ for other slip systems, i.e., there are no cross effects, including the effect of other slip systems on the loss of lattice stability along the slip system $\alpha$. Also, with the cross effects in Eq.(46), instability conditions cannot be reduced to the expected form that instability occurs in when resolved shear stress reaches some critical value. That is why we accept

\section{Condition II:}

$$
\left.\frac{\partial^{2} \psi^{l}\left(\boldsymbol{E}_{e}, \eta_{i}=n_{i}\right)}{\partial \eta_{\alpha} \partial \eta_{k}}\right|_{\boldsymbol{E}_{e}}=0 ; \quad \forall k \neq \alpha .
$$

Note that condition (47) does not impose a constraint on the cross derivative for incomplete

dislocations. With Condition II, the matrix $\left.\frac{\partial X_{\alpha}}{\partial \eta_{k}}\right|_{\boldsymbol{P}}$ becomes diagonal and the instability condition Eq.(46) simplifies to non-negativeness of any of the diagonal terms:

$$
\frac{1}{\rho_{0}} \boldsymbol{P}^{T} \cdot \boldsymbol{F}_{e}: \frac{\partial^{2} \boldsymbol{F}_{p}\left(\eta_{\alpha}=n_{\alpha}\right)}{\partial \eta_{\alpha}^{2}}-\left.\frac{\partial^{2} \psi^{l}\left(\boldsymbol{E}_{e}, \eta_{i}=n_{i}\right)}{\partial \eta_{\alpha}^{2}}\right|_{\boldsymbol{E}_{e}} \geq 0
$$

or allowing for the expression for plastic work:

$$
\frac{1}{\rho_{0}} \tau_{\alpha} \gamma_{\alpha} \frac{d^{2} \Phi\left(\eta_{\alpha}=n_{\alpha}\right)}{d \eta_{\alpha}^{2}} \geq\left.\frac{\partial^{2} \psi^{l}\left(\boldsymbol{E}_{e}, \eta_{i}=n_{i}\right)}{\partial \eta_{\alpha}^{2}}\right|_{\boldsymbol{E}_{e}} .
$$




\subsection{Initial choice of function $\Phi\left(\eta_{\alpha}\right)$ for the Burgers vector}

Function $\Phi\left(\eta_{\alpha}\right)$ has to satisfy the conditions $\Phi(n)=n$ and $\frac{d \Phi(n)}{d \eta_{\alpha}}=0$ (see Eq.(40)). For a single dislocation, these conditions for $n=0$ and $n=1$ coincide with those for the transformation strain for an austenite-martensite phase transformation (Levitas et al. (2003); Levitas (2013b)). Thus, with fourth- or sixth-degree polynomials, functions

$$
\begin{array}{r}
\Phi_{4}=a \eta_{\alpha}^{2}\left(1-\eta_{\alpha}\right)^{2}+\left(4 \eta_{\alpha}^{3}-3 \eta_{\alpha}^{4}\right) \\
\Phi_{6}=a \eta_{\alpha}^{2} / 2+(3-a) \eta_{\alpha}^{4}+(a-4) \eta_{\alpha}^{6} / 2 ; \quad 0<a<6
\end{array}
$$

where $a$ is a parameter, satisfy our conditions. To satisfy the same conditions for $n$ dislocations, we present

$$
\eta_{\alpha}=\operatorname{Int}\left(\eta_{\alpha}\right)+\bar{\eta}_{\alpha}
$$

as the sum of the integer part $\operatorname{Int}\left(\eta_{\alpha}\right)$ and the fractional part $\bar{\eta}_{\alpha}:=\eta_{\alpha}-\operatorname{Int}\left(\eta_{\alpha}\right)$. Then we define with fourth or sixth-degree polynomials, functions

$$
\begin{gathered}
\Phi_{4}=\operatorname{Int}\left(\eta_{\alpha}\right)+a \bar{\eta}_{\alpha}^{2}\left(1-\bar{\eta}_{\alpha}\right)^{2}+\left(4 \bar{\eta}_{\alpha}^{3}-3 \bar{\eta}_{\alpha}^{4}\right) \\
\Phi_{6}=\operatorname{Int}\left(\eta_{\alpha}\right)+a \bar{\eta}_{\alpha}^{2} / 2+(3-a) \bar{\eta}_{\alpha}^{4}+(a-4) \bar{\eta}_{\alpha}^{6} / 2 .
\end{gathered}
$$

While each of the terms in Eq.(52) is discontinuous along with its first derivative at points $\eta_{\alpha}=n$, functions $\Phi_{4}$ and $\Phi_{6}$ and their first derivatives are continuous at $\eta_{\alpha}=n$ and satisfy all our conditions. Further constrains will be discussed below.

\section{Helmholtz free energy}

There are several types of interactions between dislocations, each of them is described by corresponding energy. We will use the usual decomposition of the free energy per unit mass $\psi\left(\boldsymbol{E}_{e}, \eta_{\alpha}, \nabla \eta_{\alpha}\right)=\psi^{l}\left(\boldsymbol{E}_{e}, \eta_{\alpha}\right)+\psi^{\nabla}\left(\eta_{\alpha}, \nabla \eta_{\alpha}\right)$ into the local energy $\psi^{l}$ and the gradient-related 
$\psi^{\nabla}$ parts. We accept the free energy in the following form

$$
\begin{aligned}
& \psi=\psi^{e}\left(\boldsymbol{E}_{e}\right)+\psi^{c}+\psi^{i n t}+\psi^{\nabla} ; \\
& \rho_{0} \psi^{c}=\sum_{\alpha=1}^{n} A_{\alpha} \bar{\eta}_{\alpha}^{2}\left(1-\bar{\eta}_{\alpha}\right)^{2} ; \\
& \rho_{0} \psi^{i n t}=\sum_{\alpha}^{n} \sum_{k=1}^{n} \bar{A}_{\alpha k} \bar{\eta}_{\alpha}^{2}\left(1-\bar{\eta}_{\alpha}\right)^{2} \bar{\eta}_{k}^{2}\left(1-\bar{\eta}_{k}\right)^{2} ; \quad A_{\alpha \alpha}=0 ; \\
& \rho_{0} \psi^{\nabla}=\frac{\beta}{2} \sum_{\alpha=1}^{n}\left(\left|\nabla_{0} \bar{\eta}_{\alpha}\right|^{2}+\left[M\left(1-\bar{\eta}_{\alpha}\right)^{2}-1\right]\left(\nabla_{0} \bar{\eta}_{\alpha} \cdot \boldsymbol{n}_{0}^{\alpha}\right)^{2}\right) .
\end{aligned}
$$

where $\psi^{e}, \psi^{c}$, and $\psi^{\text {int }}$ are the elastic and crystalline energies, as well as the energy of the interaction of dislocation cores belonging to different slip systems, respectively. Since $\eta_{\alpha}=$ $\bar{\eta}_{\alpha}+\operatorname{Int}\left(\eta_{\alpha}\right)$, then $\nabla_{0} \bar{\eta}_{\alpha}=\nabla_{0} \eta_{\alpha}$ and bar above $\eta_{\alpha}$ can be omitted when gradients are evaluated.

Elastic energy. As usual, we assume that the elastic energy $\psi^{e}\left(\boldsymbol{E}_{e}\right)$ is independent of $\eta_{\alpha}$ for fixed elastic strain $\boldsymbol{E}_{e}$, i.e., elastic constants in the region of the dislocation core are the same as in a perfect lattice. Should such information be available, it can easily be included in the elastic constants. Since dislocations do not change the crystal lattice, the elastic energy is independent of the order parameters and can be accepted as the Taylor series of the elastic Lagrangian strain

$$
\rho_{0} \psi^{e}\left(\boldsymbol{E}_{e}\right)=\frac{1}{2} \boldsymbol{E}_{e}: \boldsymbol{C}: \boldsymbol{E}_{e}+\frac{1}{3 !}\left(\boldsymbol{E}_{e}: \boldsymbol{C}^{3}: \boldsymbol{E}_{e}\right): \boldsymbol{E}_{e}+\frac{1}{4 !} \boldsymbol{E}_{e}:\left(\boldsymbol{E}_{e}: \boldsymbol{C}^{4}: \boldsymbol{E}_{e}\right): \boldsymbol{E}_{e}+\ldots
$$

where $C^{k}$ are the elastic moduli tensors of the $k^{t h}$ rank. Using Eq.(36) and the fact that $\frac{\partial \psi}{\partial \boldsymbol{F}_{e}}=\boldsymbol{F}_{e} \cdot \frac{\partial \psi}{\partial \boldsymbol{E}_{e}}$, the elasticity rule Eq.( 30$)$ can be expressed as following:

$$
\boldsymbol{P}=\rho_{0} \boldsymbol{F}_{e} \cdot \frac{\partial \psi^{e}}{\partial \boldsymbol{E}_{e}} \cdot \boldsymbol{F}_{p}^{T-1} ; \quad \boldsymbol{\sigma}=\frac{\rho_{0}}{J} \boldsymbol{F}_{e} \cdot \frac{\partial \psi^{e}}{\partial \boldsymbol{E}_{e}} \cdot \boldsymbol{F}_{p}^{T-1} \cdot \boldsymbol{F}_{p}^{T} \cdot \boldsymbol{F}_{e}^{T}=\frac{\rho_{0}}{J} \boldsymbol{F}_{e} \cdot \frac{\partial \psi^{e}}{\partial \boldsymbol{E}_{e}} \cdot \boldsymbol{F}_{e}^{T}
$$

with

$$
\rho_{0} \frac{\partial \psi^{e}}{\partial \boldsymbol{E}_{e}}=\boldsymbol{C}: \boldsymbol{E}_{e}+\frac{1}{2}\left(\boldsymbol{E}_{e}: \boldsymbol{C}^{3}: \boldsymbol{E}_{e}\right)+\frac{1}{3 !}\left(\boldsymbol{E}_{e}: \boldsymbol{C}^{4}: \boldsymbol{E}_{e}\right): \boldsymbol{E}_{e}+\ldots
$$

In our applications, we will use the simplest function which contains only the first term both in Eq.(57) and (59). Due to generation of elastic stresses (Eq.(58)) and their contribution to the driving force for dislocation evolution Eq.(31), elastic energy produces the major interaction between dislocations, similar to discrete dislocation model. In combination with phase field kinematic description of dislocations, it determines, in particular, repulsion between dislocations in the same plane, repulsion or attraction of dislocations in different slip systems, 
annihilation and multiplication of dislocations, and Lomer lock formation. It comes directly from the coupled solution of the phase field and mechanics equations and is a part of any PFA to dislocations. However, our approach produces different elastic interaction due to different interpolation function $\Phi$ for Burgers vector in Eq.(10) and different stress field, because of large strain formulation, nonlinear elasticity, and also different boundary conditions (see Section 6). Remark. It looks confusing that the elastic energy produces the major interaction between dislocations and affects dislocation evolution but the elastic energy $\psi^{e}\left(\boldsymbol{E}_{e}\right)$ is independent of $\eta_{\alpha}$ and does not contribute to the Ginzburg-Landau Eq.(31). This confusion can be resolved by considering different strain-related arguments of the elastic energy. If $\psi^{e}$ is considered as a function of elastic strain then it is independent of $\eta_{\alpha}$, but the term with stresses appears in the Ginzburg-Landau Eq.(31) due to thermodynamic treatment, which describes the elastic interaction between dislocations. If we utilize $\boldsymbol{F}_{e}=\boldsymbol{F} \cdot \boldsymbol{F}_{p}{ }^{-1}$ and substitute it in $\psi^{e}\left(\boldsymbol{F}_{e}\right)=\psi^{e}\left(\boldsymbol{F} \cdot \boldsymbol{F}_{p}{ }^{-1}\right)=\tilde{\psi}^{e}\left(\boldsymbol{F}, \boldsymbol{F}_{p}\right)$, then at fixed $\boldsymbol{F}$ the elastic energy depends on $\eta_{\alpha}$ through $\boldsymbol{F}_{p}$ and consequently contributes to the Ginzburg-Landau equation. However, when $\boldsymbol{F}$ is used as an argument of free energy instead of $\boldsymbol{F}_{e}$, there is no direct contribution of stresses to the Ginzburg-Landau Eq.(31). Of course both presentations lead to the same results (see, e.g., approach in terms of the total deformation gradient $\boldsymbol{F}$ in Levitas (2014)), i.e., both changes to Eq.(31) compensate each other.

Crystalline energy. The crystalline (Peierls) energy is a periodic function of each order parameter and is usually described in terms of trigonometric functions. Following Levitas et al. (2003), we will use the simplest double well function (54) used in phase transformation theory, periodically continued with the help of the fractional part of the order parameter. This function demonstrates good correspondence with molecular dynamic simulations for the gamma surface (Lee et al. (2011)). Assuming here $\psi^{\text {int }}=0$, we demonstrate that such crystalline energy satisfies all the formulated conditions. Indeed, condition (40)

$$
\frac{\partial \psi^{l}\left(\boldsymbol{E}_{e}, \eta_{i}=n_{\beta}\right)}{\partial \eta_{\alpha}}=2 A_{\alpha} \bar{\eta}_{\alpha}\left(1-\bar{\eta}_{\alpha}\right)\left(1-2 \bar{\eta}_{\alpha}\right)=0 .
$$

is satisfied for $\bar{\eta}_{\alpha}=0$ and $\bar{\eta}_{\alpha}=1$, i.e., for $\eta_{\alpha}=n$ independent of a number of dislocations along other slip systems.

The thermodynamic equilibrium condition Eq.(39) with function $\Phi_{4}$ results (in addition to the above stable solutions) in the third unstable solution for $\bar{\eta}_{\alpha}$, which can be presented in the 
form of the resolved shear stress - order parameter curve:

$$
\tau_{\alpha}=\frac{A_{\alpha}}{\gamma_{\alpha}} \frac{\left(1-2 \bar{\eta}_{\alpha}\right)}{a\left(1-2 \bar{\eta}_{\alpha}\right)+6 \bar{\eta}_{\alpha}} .
$$

Substituting in Eq.(61) $\bar{\eta}_{\alpha}=0$, we obtain the critical shear stress to nucleate the $(n+1)^{t h}$ dislocation: $\tau_{\alpha}=\frac{A_{\alpha}}{a \gamma_{\alpha}}$. Substituting in Eq.(61) $\bar{\eta}_{\alpha}=1$, we obtain the critical shear stress to nucleate the dislocation in the opposite direction, i.e., from $n$ to $(n-1)$ dislocations: $\tau_{\alpha}=$ $-\frac{A_{\alpha}}{(6-a) \gamma_{\alpha}}$. Since the magnitude of the critical shear stress for the nucleation of positive and negative dislocations is supposed to be the same, we conclude that $a=3$, similar to twinning in Levitas and Preston (2002b); Levitas and Javanbakht (2011a). Note that the critical shear stress coincides with that obtained from the instability condition Eq.(49) for $\bar{\eta}_{\alpha}=0$ and $\bar{\eta}_{\alpha}=1$. Summarizing for $a=3$, one obtains:

$$
\Phi=\phi\left(\bar{\eta}_{\alpha}\right)+\operatorname{Int}\left(\eta_{\alpha}\right) ; \quad \phi\left(\bar{\eta}_{\alpha}\right)=\bar{\eta}_{\alpha}^{2}\left(3-2 \bar{\eta}_{\alpha}\right)
$$

the shear stress - order parameter curve:

$$
\tau_{\alpha}=\frac{A_{\alpha}}{3 \gamma_{\alpha}}\left(1-2 \bar{\eta}_{\alpha}\right)
$$

and the instability conditions:

$$
n \rightarrow n+1: \quad \tau_{\alpha} \geq \frac{A_{\alpha}}{3 \gamma_{\alpha}} ; \quad n \rightarrow n-1: \quad \tau_{\alpha} \leq-\frac{A_{\alpha}}{3 \gamma_{\alpha}} .
$$

Note that for a single slip system, the sixth-degree function $\Phi_{6}$ combined with the sixth-degree crystalline energy $\rho_{0} \psi^{c}=\sum_{\alpha=1}^{n} A_{\alpha} \bar{\eta}_{\alpha}^{2}\left(1-\bar{\eta}_{\alpha}^{2}\right)^{2}$ has been suggested in Levitas et al. (2003). While for $a=3$ it results in the same lattice instability condition (64), both the crystalline energy and the shear stress - order parameter curve are not symmetric with respect to the exchange of $\bar{\eta}_{\alpha}$ and $1-\bar{\eta}_{\alpha}$, in contrast to the fourth-degree polynomial. Since such a symmetry is expected for lattices for which positive and negative slips are equivalent, we will focus below on the fourth-degree polynomial only.

For all equivalent slip systems $A_{\alpha}$ is the same. To introduce the height $H^{\alpha}$ of the dislocation bands through equations rather than through a computational mesh, the coefficient $A_{\alpha}$ is chosen to be a periodic step-wise function of the coordinate $y^{\alpha}$ along the normal to the slip 
plane $\boldsymbol{n}_{0}^{\alpha}$ :

$$
\begin{aligned}
& \bar{y}^{\alpha}=y^{\alpha}-\operatorname{Int}\left(\frac{y^{\alpha}}{H^{\alpha}+w_{\alpha}}\right)\left(H^{\alpha}+w_{\alpha}\right) ; \\
& A_{\alpha}\left(y^{\alpha}\right)= \begin{cases}q A_{\alpha}^{0} & \bar{y}^{\alpha}>H^{\alpha} \\
A_{\alpha}^{0} & \bar{y}^{\alpha} \leq H^{\alpha}\end{cases}
\end{aligned}
$$

Thus, the parameter $A_{\alpha}$ is equal to its normal value within each dislocation band of the height $H^{\alpha}$ and $q A_{\alpha}^{0}(q \gg 1)$ in a thin boundary layer between dislocations of the width $w_{\alpha}=p H^{\alpha}$ $(p \ll 1)$ (Fig. 2). Large values of $A_{\alpha}$ prevent the widening of the dislocation outside the designated dislocation band.
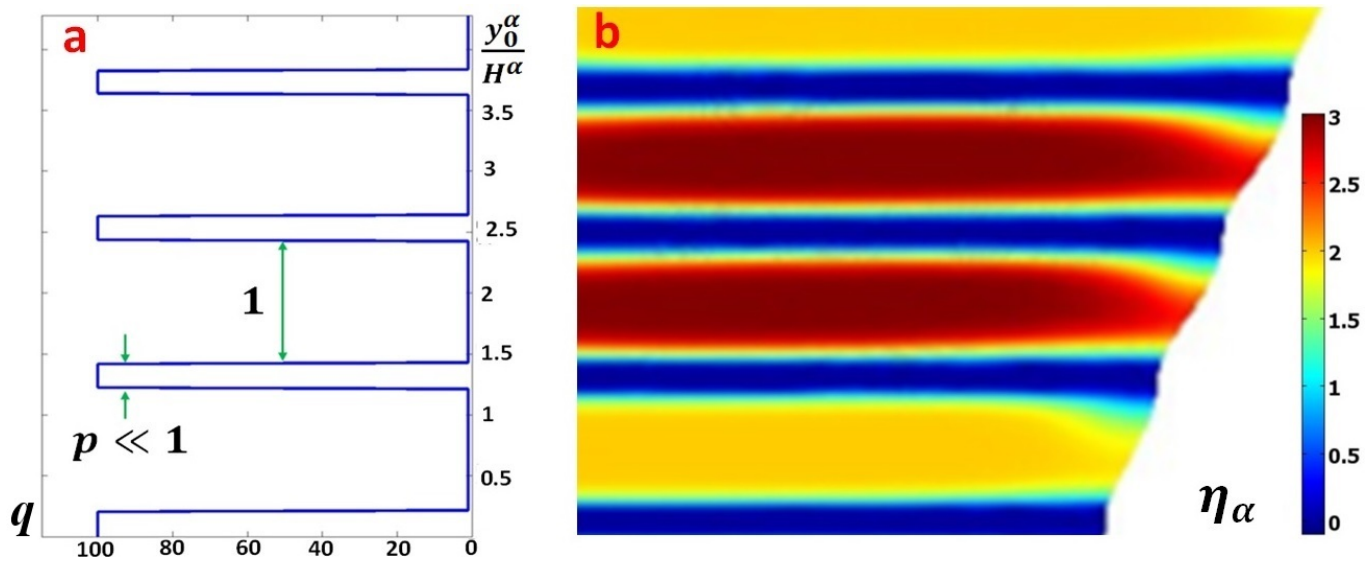

Figure 2: (a) Schematics of the distribution of the multiplier $q$ for the crystalline energy barrier $A_{\alpha}=q A_{\alpha}^{0}$ along the normal to each slip plane. It represents a periodic step-wise function with $q=1$ within the slip band and $q=100$ in a thin boundary layer between dislocations of the width $w_{\alpha}=p H^{\alpha}(p \ll 1)$. (b) An exemplary distribution of the dislocation order parameter $\eta_{\alpha}$ for the corresponding distribution of the multiplier $q$.

Energy of the interaction of cores of different dislocations. PFA for multivariant martensitic phase transformations (Jin et al. (2001); Chen (2002); Wang and Khachaturyan (1997); Artemev et al. (2001); Levitas and Preston (2002b); Levitas et al. (2003)) contains cross terms $\eta_{\alpha} \eta_{k}$, which mimic and penalize the interaction of different martensitic variants through structural variables (order parameters), in addition to their interaction through the stress field. Similar cross terms were absent in the previous dislocation theories (Wang et al. (2001a,c); Koslowski et al. (2002); Koslowski and Ortiz (2004); Rodney et al. (2003); Wang and Li (2010); Hunter et al. (2010); Wang and Shen (2003); Levitas and Javanbakht (2012, 2013, 2014)). Here we introduced such a cross terms $\psi^{\text {int }}$ to explore their possible structure and significance for dislocations. According to Eq.(55), this energy penalizes the interaction of the dislocation cores of dislocations belonging to different slip systems at the same point. It depends on $\bar{\eta}_{\alpha}$ rather than $\eta_{\alpha}$, because after a complete dislocation has passed through the point under con- 
sideration, it does not affect the crystal structure at this point. Self-interaction is excluded by imposing $A_{\alpha \alpha}=0$. Thus, when just one incomplete dislocation is present in the given point, this term disappears. The coefficients $\bar{A}_{\alpha k}$ are the same for equivalent dislocation pairs. The interaction energy should be symmetric with respect to the exchange of any $\eta_{\alpha}$ with $1-\eta_{\alpha}$ and subscripts $\alpha$ with $k$, which can be satisfied by using Eq.(55) with the same exponents for all four multipliers. Since without the interaction term all our conditions are satisfied, $\psi^{\text {int }}$ is chosen as the minimum degree polynomial that does not violate any of them. Thus, the exponent 2 is the minimal integer exponent for which $\psi^{\text {int }}$ and its first and second derivatives (including the mixed one) are zero when either $\bar{\eta}_{\alpha}=0$ or 1 or $\bar{\eta}_{k}=0$ or 1 . Indeed, $\psi^{\text {int }}$ and its derivatives

$$
\begin{aligned}
& \frac{\partial \psi^{i n t}}{\partial \bar{\eta}_{\alpha}}=2 \bar{A}_{\alpha k} \bar{\eta}_{\alpha}\left(1-\bar{\eta}_{\alpha}\right)\left(1-2 \bar{\eta}_{\alpha}\right) \bar{\eta}_{k}^{2}\left(1-\bar{\eta}_{k}\right)^{2} ; \\
& \frac{\partial^{2} \psi^{i n t}}{\partial^{2} \bar{\eta}_{\alpha}}=2 \bar{A}_{\alpha k}\left(1-6 \bar{\eta}_{\alpha}+6 \bar{\eta}_{\alpha}^{2}\right) \bar{\eta}_{k}^{2}\left(1-\bar{\eta}_{k}\right)^{2} ; \\
& \frac{\partial^{2} \psi^{i n t}}{\partial \bar{\eta}_{\alpha} \partial \bar{\eta}_{k}}=4 \bar{A}_{\alpha k} \bar{\eta}_{\alpha}\left(1-\bar{\eta}_{\alpha}\right)\left(1-2 \bar{\eta}_{\alpha}\right) \bar{\eta}_{k}\left(1-\bar{\eta}_{k}\right)\left(1-2 \bar{\eta}_{k}\right) .
\end{aligned}
$$

satisfy these conditions. If, however, the exponents in all multipliers in $\psi^{\text {int }}$ are equal to 1 , the interaction term will spoil the instability condition because the mixed derivative of $\psi^{\text {int }}$

$$
\frac{\partial^{2} \psi^{i n t}}{\partial \bar{\eta}_{\alpha} \partial \bar{\eta}_{k}}=\bar{A}_{\alpha k}\left(1-2 \bar{\eta}_{\alpha}\right)\left(1-2 \bar{\eta}_{k}\right) .
$$

is not zero neither for $\bar{\eta}_{\alpha}=0$ or 1 nor for $\bar{\eta}_{k}=0$ and 1 . This violates Condition II.

As it is shown in simulations in Javanbakht and Levitas (2014), $\psi^{\text {int }}$ affects evolution of dislocations when they move along the intersection of slip systems. Whether this effect corresponds to reality and what should be the value of the parameter $\bar{A}_{\alpha k}$ for different materials should be determined by comparison of PFA and atomistic simulations.

Gradient energy. In general for dislocations, the gradient energy is localized at the dislocation core region, thus contributing to the core energy. We decompose

$$
\nabla_{0} \eta_{\alpha}=\nabla_{0}^{m} \eta_{\alpha} \boldsymbol{m}_{0}^{\alpha}+\nabla_{0}^{n} \eta_{\alpha} \boldsymbol{n}_{0}^{\alpha} ; \quad \nabla_{0}^{m} \eta_{\alpha}:=\nabla_{0} \eta_{\alpha} \cdot \boldsymbol{m}_{0}^{\alpha} ; \quad \nabla_{0}^{n} \eta_{\alpha}:=\nabla_{0} \eta_{\alpha} \cdot \boldsymbol{n}_{0}^{\alpha}
$$

into contributions along the slip direction and along the direction normal to the slip plane. It is generally accepted that free energy should be independent of the contribution $\nabla_{0}^{n} \eta_{\alpha}$ along the direction normal to the slip plane, because slip planes represent a perfect lattice and do not possess any dislocation-related energy away from the dislocation cores (Shen and Wang 
(2004); Wang et al. (2001b); Jin and Khachaturyan (2001); Wang et al. (2003); Hu et al. (2004); Koslowski et al. (2002); Koslowski and Ortiz (2004); Wang and Li (2010); Kundin et al. (2011)). This distinguishes gradient energy of dislocations from that for martensitic units and twins, because phase and twin interfaces do possess energy. As we mentioned in Introduction, this, however, leads to the lack of a characteristic length in the directions $\boldsymbol{n}_{0}^{\alpha}$, an ill-posed system of evolution equations for $\eta_{\alpha}$, and a catastrophic mesh-dependence of the solution. Thus, one has to introduce a characteristic length in the directions $\boldsymbol{n}_{0}^{\alpha}$ and regularize the problem, still keeping the energy of the interface $\Sigma$ between the complete dislocation band and the rest of the crystal zero. In the approximation of the quadratic form, the simplest expression is

$$
\rho_{0} \psi^{\nabla}=0.5 \sum_{\alpha=1}^{p} \sum_{\varsigma=1}^{p}\left(\beta_{\alpha}^{\varsigma} \nabla_{0}^{m} \eta_{\alpha} \nabla_{0}^{m} \eta_{\varsigma}+M\left(1-\bar{\eta}_{\alpha}\right)^{2} \nu_{\alpha}^{\varsigma} \nabla_{0}^{n} \eta_{\alpha} \nabla_{0}^{n} \eta_{\varsigma}\right) .
$$

Assuming the same gradient coefficients along the slip planes and also the same gradient coefficients normal to the slip planes for all slip systems, as well as an absence of the crosseffects, the gradient energy Eq.(71) can be simplified into the gradient energy $\psi^{\nabla}$ in Eq.(53), which was suggested in Levitas and Javanbakht (2012). If $M=0$, the gradient energy in Eq.(53) coincides with known expressions (Wang et al. (2001b); Jin and Khachaturyan (2001); Wang et al. (2003); Hu et al. (2004)). However, for $M=0$, after nucleation, the dislocation propagates within a band of one finite element high, which is unphysical and the results are mesh-dependent. An additional term with $M \ll 1$ penalizes gradients along the normal $\boldsymbol{n}_{0}^{\alpha}$, which leads to dislocation propagation within the entire band of the height $H^{\alpha}$. This term disappears when the dislocation is completed $\left(\bar{\eta}_{\alpha}=1\right)$, and does not produce artificial surface energy at the boundary $\Sigma$. Still, it makes some contribution to the energy of the dislocation core, which will be taken into account during the calibration of material parameters. It is shown in Levitas and Javanbakht (2012) that the value $M=0.1$ leads to proper regularization and desired solution.

\section{Ginzburg-Landau equations}

It is traditional in nonequilibrium thermodynamics that one has to assume a general, nonlinear kinetic equation $\dot{\eta}_{\alpha}=f\left(X_{i}\right)$ connecting the slip rate in the $\alpha^{\text {th }}$ slip system with the driving force for the $i^{\text {th }}$ force-i.e., including cross effects. In the linear approximation $\dot{\eta}_{\alpha}=L_{\alpha}^{i} X_{i}$, $L_{\alpha}^{i}$ are positive definite kinetic coefficients, which satisfy the Onsager reciprocal relationships 
$L_{\alpha}^{i}=L_{i}^{\alpha}$. Using Eq.(31) for the thermodynamic force conjugate to $\dot{\eta}_{\alpha}$, and assuming the same kinetics coefficient for all slip systems, i.e. $\dot{\eta}_{\alpha}=L X_{\alpha}$, we can write the Ginzburg-Landau equation as

$$
\frac{1}{L} \frac{\partial \eta_{\alpha}}{\partial t}=\frac{1}{\rho_{0}} \tau_{\alpha} \gamma_{\alpha} \frac{d \Phi}{d \eta_{\alpha}}-\frac{\partial \psi}{\partial \eta_{\alpha}}+\frac{1}{\rho_{0}} \nabla_{0} \cdot\left(\rho_{0} \frac{\partial \psi}{\partial \nabla_{0} \eta_{\alpha}}\right) .
$$

If $\rho_{0}$ is homogeneous, then the terms with $\rho_{0}$ eliminate each other in the last term of Eq.(72). Utilizing the expression

$$
\frac{1}{\rho_{0}} \nabla_{0} \cdot\left(\rho_{0} \frac{\partial \psi}{\partial \nabla_{0} \eta}\right)=\frac{1}{\rho} \nabla \cdot\left(\rho \frac{\partial \psi}{\partial \boldsymbol{\nabla} \eta}\right) .
$$

from Appendix, the Ginzburg-Landau equations (72) can be transformed to the actual configuration

$$
\frac{1}{L} \frac{D \eta_{\alpha}(\boldsymbol{r}, t)}{D t}=\frac{1}{L}\left(\frac{\partial \eta_{\alpha}(\boldsymbol{r}, t)}{\partial t}+\boldsymbol{v} \cdot \nabla \eta_{\alpha}\right)=\frac{1}{\rho_{0}} \tau_{\alpha} \gamma_{\alpha} \frac{d \Phi}{d \eta_{\alpha}}-\frac{\partial \psi}{\partial \eta_{\alpha}}+\frac{1}{\rho} \boldsymbol{\nabla} \cdot\left(\rho \frac{\partial \psi}{\partial \boldsymbol{\nabla} \eta_{\alpha}}\right) .
$$

We substituted the material time derivative of $\eta_{\alpha}$ in the reference configuration with the corresponding expression in the current configuration but did not change the first two terms in Eq.(74), because they are defined per unit mass and are the same in any configuration. Since, when the resolved shear stress is defined in terms of the Cauchy stress (see Eq.(37)) it is proportional to $J$, by introducing resolved shear stress $\tilde{\tau}_{\alpha}:=\tau_{\alpha} / J$ one can transform the plastic work in Eq.(74) to $\frac{1}{\rho} \tilde{\tau}_{\alpha} \gamma_{\alpha} \frac{d \Phi}{d \eta_{\alpha}}$ and make Eq.(74) more consistent in terms of using $\rho$ in the current configuration.

Let us obtain a more detailed form of the Ginzburg-Landau Eq.(72) utilizing Eqs.(53)Eq.(56). Since the elastic energy $\psi^{e}$ is independent of $\eta_{\alpha}$, it does not contribute to the GinzburgLandau equation. The contribution from the local derivative with respect to $\eta_{\alpha}$ is

$$
\begin{aligned}
& \frac{\partial \psi}{\partial \eta_{\alpha}}=\frac{2}{\rho_{0}} A_{\alpha} \bar{\eta}_{\alpha}\left(1-\bar{\eta}_{\alpha}\right)\left(1-2 \bar{\eta}_{\alpha}\right)+\frac{2}{\rho_{0}} \bar{\eta}_{\alpha}\left(1-\bar{\eta}_{\alpha}\right)\left(1-2 \bar{\eta}_{\alpha}\right) \sum_{k=1}^{n} \bar{A}_{\alpha k} \bar{\eta}_{k}^{2}\left(1-\bar{\eta}_{k}\right)^{2} \\
& -\frac{1}{\rho_{0}} \beta\left(M\left(1-\bar{\eta}_{\alpha}\right)\left(\nabla_{0} \bar{\eta}_{\alpha} \cdot \boldsymbol{n}_{0}^{\alpha}\right)^{2}\right) .
\end{aligned}
$$

Now, let us elaborate the last term in Ginzburg-Landau Eq.(72) related to the gradient term $\psi^{\nabla}$

$$
\frac{\partial \psi^{\nabla}}{\partial \nabla_{0} \eta_{\alpha}}=\frac{1}{\rho_{0}} \beta\left(\nabla_{0} \bar{\eta}_{\alpha}+\left[M\left(1-\bar{\eta}_{\alpha}\right)^{2}-1\right]\left(\nabla_{0} \bar{\eta}_{\alpha} \cdot \boldsymbol{n}_{0}^{\alpha}\right) \boldsymbol{n}_{0}^{\alpha}\right) .
$$

Applying the divergence operator $\nabla_{0}$. to Eq.(76) and taking into account that $\boldsymbol{n}_{0}^{\alpha}$ does not 
vary in space (and in time), one obtains

$$
\begin{aligned}
& \nabla_{0} \cdot\left(\rho_{0} \frac{\partial \psi^{\nabla}}{\partial \nabla_{0} \eta_{\alpha}}\right)= \\
& \beta\left(\nabla_{0}^{2} \bar{\eta}_{\alpha}-2 M\left(1-\bar{\eta}_{\alpha}\right)\left(\nabla_{0} \bar{\eta}_{\alpha} \cdot \boldsymbol{n}_{0}^{\alpha}\right)^{2}+\left[M\left(1-\bar{\eta}_{\alpha}\right)^{2}-1\right]\left(\boldsymbol{n}_{0}^{\alpha} \cdot \nabla_{0}\right)\left(\nabla_{0} \bar{\eta}_{\alpha} \cdot \boldsymbol{n}_{0}^{\alpha}\right)\right) .
\end{aligned}
$$

In a component form, the last term is

$$
\left(\boldsymbol{n}_{0}^{\alpha} \cdot \nabla_{0}\right)\left(\nabla_{0} \bar{\eta}_{\alpha} \cdot \boldsymbol{n}_{0}^{\alpha}\right)=\frac{\partial^{2} \bar{\eta}_{\alpha}}{\partial r_{0 i} \partial r_{0 k}} n_{0 i}^{\alpha} n_{0 k}^{\alpha}
$$

Substituting Eqs.(75) and (77) in Eq.(72), the Ginzburg-Landau in the reference configuration specifies as

$$
\begin{aligned}
& \frac{1}{L} \frac{\partial \eta_{\alpha}}{\partial t}=\frac{1}{\rho_{0}} \tau_{\alpha} \gamma_{\alpha} \frac{d \Phi}{d \eta_{\alpha}}-\frac{2}{\rho_{0}} A_{\alpha} \bar{\eta}_{\alpha}\left(1-\bar{\eta}_{\alpha}\right)\left(1-2 \bar{\eta}_{\alpha}\right) \\
& -\frac{2}{\rho_{0}} \bar{\eta}_{\alpha}\left(1-\bar{\eta}_{\alpha}\right)\left(1-2 \bar{\eta}_{\alpha}\right) \sum_{k=1}^{n} \bar{A}_{\alpha k} \bar{\eta}_{k}^{2}\left(1-\bar{\eta}_{k}\right)^{2} \\
& +\beta\left(\nabla_{0}^{2} \bar{\eta}_{\alpha}-2 M\left(1-\bar{\eta}_{\alpha}\right)\left(\nabla_{0} \bar{\eta}_{\alpha} \cdot \boldsymbol{n}_{0}^{\alpha}\right)^{2}+\left[M\left(1-\bar{\eta}_{\alpha}\right)^{2}-1\right]\left(\boldsymbol{n}_{0}^{\alpha} \cdot \nabla_{0}\right)\left(\nabla_{0} \bar{\eta}_{\alpha} \cdot \boldsymbol{n}_{0}^{\alpha}\right)\right) .
\end{aligned}
$$

In order to transform Eq.(79) to the actual configuration, we recall that

$$
\frac{\partial \eta_{\alpha}}{\partial r_{0 i}}=\frac{\partial \eta_{\alpha}}{\partial r_{k}} \frac{\partial r_{k}}{\partial r_{0 i}}=\frac{\partial \eta_{\alpha}}{\partial r_{k}} F_{k i} ; \quad \nabla_{0} \eta_{\alpha}=\nabla \eta_{\alpha} \cdot \boldsymbol{F}=\boldsymbol{F}^{T} \cdot \nabla \eta_{\alpha}
$$

and introduce the normal to the slip plane in the actual configuration, $\boldsymbol{n}^{\alpha}=\boldsymbol{n}_{0}^{\alpha} \cdot \boldsymbol{F}_{e}^{-1}$ (Asaro (1983); Havner (1992); Lubarda (2002)), which, however, does not have a unit length. Then

$$
\begin{aligned}
& \boldsymbol{n}_{0}^{\alpha}=\boldsymbol{n}^{\alpha} \cdot \boldsymbol{F}_{e} ; \quad \boldsymbol{n}_{0}^{\alpha} \cdot \boldsymbol{\nabla}_{0}=\boldsymbol{n}^{\alpha} \cdot \boldsymbol{F}_{e} \cdot \boldsymbol{F}^{T} \cdot \boldsymbol{\nabla} ; \\
& \boldsymbol{\nabla}_{0} \bar{\eta}_{\alpha} \cdot \boldsymbol{n}_{0}^{\alpha}=\nabla \bar{\eta}_{\alpha} \cdot \boldsymbol{F} \cdot \boldsymbol{F}_{e}^{T} \cdot \boldsymbol{n}^{\alpha}
\end{aligned}
$$

According to Eq.s (112) and (115) in Appendix, one has

$$
\nabla_{0}^{2} \bar{\eta}_{\alpha}=\nabla_{0} \cdot \nabla_{0} \bar{\eta}_{\alpha}=(\boldsymbol{\nabla} \cdot \boldsymbol{F}) \cdot\left(\boldsymbol{\nabla} \bar{\eta}_{\alpha} \cdot \boldsymbol{F}\right)=\left(\boldsymbol{F}^{T} \cdot \boldsymbol{\nabla}\right) \cdot\left(\boldsymbol{\nabla} \bar{\eta}_{\alpha} \cdot \boldsymbol{F}\right)=\left(\boldsymbol{F} \cdot \boldsymbol{F}^{T}\right): \nabla \nabla \bar{\eta}_{\alpha}
$$

and

$$
\left(\boldsymbol{n}^{\alpha} \cdot \nabla\right)\left(\nabla \bar{\eta}_{\alpha} \cdot \boldsymbol{F} \cdot \boldsymbol{F}_{e}^{T} \cdot \boldsymbol{n}^{\alpha}\right)=\boldsymbol{n}^{\alpha} \cdot \boldsymbol{\nabla} \nabla \bar{\eta}_{\alpha} \cdot \boldsymbol{F} \cdot \boldsymbol{F}_{e}^{T} \cdot \boldsymbol{n}^{\alpha}
$$

i.e., for the gradient in the current configuration $\boldsymbol{\nabla}$, both the deformation gradient $\boldsymbol{F}$ and its elastic part $\boldsymbol{F}_{e}$ can be treated as constant tensors. Substituting Eqs.(81)-(83) in Eq.(79), the 
Ginzburg-Landau equation in the actual configuration can be presented as

$$
\begin{aligned}
& \frac{1}{L} \frac{\partial \eta_{\alpha}}{\partial t}=\frac{1}{\rho_{0}} \tau_{\alpha} \gamma_{\alpha} \frac{d \Phi}{d \eta_{\alpha}}-\frac{2}{\rho_{0}} A_{\alpha} \bar{\eta}_{\alpha}\left(1-\bar{\eta}_{\alpha}\right)\left(1-2 \bar{\eta}_{\alpha}\right) \\
& -\frac{2}{\rho_{0}} \bar{\eta}_{\alpha}\left(1-\bar{\eta}_{\alpha}\right)\left(1-2 \bar{\eta}_{\alpha}\right) \sum_{k=1}^{n} \bar{A}_{\alpha k} \bar{\eta}_{k}^{2}\left(1-\bar{\eta}_{k}\right)^{2} \\
& +\beta\left(\left(\boldsymbol{F} \cdot \boldsymbol{F}^{T}\right): \nabla \nabla \bar{\eta}_{\alpha}-2 M\left(1-\bar{\eta}_{\alpha}\right)\left(\nabla \bar{\eta}_{\alpha} \cdot \boldsymbol{n}^{\alpha}\right)^{2}+\left[M\left(1-\bar{\eta}_{\alpha}\right)^{2}-1\right] \boldsymbol{n}^{\alpha} \cdot \nabla \nabla \bar{\eta}_{\alpha} \cdot \boldsymbol{F} \cdot \boldsymbol{F}_{e}^{T} \cdot \boldsymbol{n}^{\alpha}\right) .
\end{aligned}
$$

The resolved shear stress Eq.(37) can be presented in terms of tensors in the actual configuration

$$
\tau_{\alpha}:=J \boldsymbol{n}^{\alpha} \cdot \boldsymbol{\sigma} \cdot \boldsymbol{m}^{\alpha} ; \quad \boldsymbol{m}^{\alpha}:=\boldsymbol{F}_{e} \cdot \boldsymbol{m}_{0}^{\alpha}
$$

For small strains and rotations, $\boldsymbol{P} \simeq \boldsymbol{\sigma}, \boldsymbol{F} \simeq \boldsymbol{F}_{p} \simeq \boldsymbol{F}_{e} \simeq \boldsymbol{I}, \tau_{\alpha}=\boldsymbol{n}^{\alpha} \cdot \boldsymbol{\sigma} \cdot \boldsymbol{m}^{\alpha}$ and GinzburgLandau equations in the reference and actual configurations coincide:

$$
\begin{aligned}
& \frac{1}{L} \frac{\partial \eta_{\alpha}}{\partial t}=\frac{1}{\rho_{0}} \tau_{\alpha} \gamma_{\alpha} \frac{d \Phi}{d \eta_{\alpha}}-\frac{2}{\rho_{0}} A_{\alpha} \bar{\eta}_{\alpha}\left(1-\bar{\eta}_{\alpha}\right)\left(1-2 \bar{\eta}_{\alpha}\right) \\
& -\frac{2}{\rho_{0}} \bar{\eta}_{\alpha}\left(1-\bar{\eta}_{\alpha}\right)\left(1-2 \bar{\eta}_{\alpha}\right) \sum_{k=1}^{n} \bar{A}_{\alpha k} \bar{\eta}_{k}^{2}\left(1-\bar{\eta}_{k}\right)^{2} \\
& +\beta\left(\nabla^{2} \bar{\eta}_{\alpha}-2 M\left(1-\bar{\eta}_{\alpha}\right)\left(\nabla \bar{\eta}_{\alpha} \cdot \boldsymbol{n}^{\alpha}\right)^{2}+\left[M\left(1-\bar{\eta}_{\alpha}\right)^{2}-1\right] \boldsymbol{n}^{\alpha} \cdot \nabla \nabla \bar{\eta}_{\alpha} \cdot \boldsymbol{n}^{\alpha}\right) .
\end{aligned}
$$

\section{Boundary conditions for order parameters}

In most papers devoted to the phase field simulations of dislocation evolution (Wang et al. (2001a,b,c); Jin and Khachaturyan (2001); Wang et al. (2003); Wang and Li (2010)) periodic boundary conditions for $\eta_{\alpha}$ are used. This is always the case when spectral methods of solutions of the boundary value problems are utilized (Wang et al. (2001a,b,c); Jin and Khachaturyan (2001); Wang et al. (2003); Wang and Li (2010)). In principle, the condition $\eta_{\alpha}=n$ can be applied that fixes a number of dislocations at the surface, if there are physical reasons for it. Having introduced generalized vector-forces $\Theta_{\alpha}^{0}$ for dislocation order parameters $\eta_{\alpha}$ at the external surface, one can prescribe their normal component at the boundary, similar to the heat flux:

$$
\boldsymbol{k}_{0} \cdot \boldsymbol{\Theta}_{\alpha}^{0}=\boldsymbol{k}_{0} \cdot \rho_{0} \frac{\partial \psi}{\partial \nabla_{0} \eta_{\alpha}}=Q_{\alpha}^{0}
$$

where $Q_{\alpha}^{0}$ are some given functions. In particular, for phase transformations the following type 
of boundary conditions are used (Lipowsky (1982); Pluis et al. (1990); Levitas and Javanbakht (2010); Levitas and Samani (2011a,b))

$$
\boldsymbol{k}_{0} \cdot \rho_{0} \frac{\partial \psi}{\partial \nabla_{0} \eta_{\alpha}}=-\frac{\partial \gamma_{0 s}\left(\eta_{\alpha}\right)}{\partial \eta_{\alpha}} .
$$

where $\gamma_{0 s}\left(\eta_{\alpha}\right)$ is the surface energy per unit reference area. For phase transformations, if surface energy is different for the parent and product phases, then it should depend on the order parameters. This leads to surface-induced pre-transformations and barrierless nucleation (Lipowsky (1982); Pluis et al. (1990); Levitas and Javanbakht (2010); Levitas and Samani $(2011 \mathrm{a}, \mathrm{b}))$. For dislocations surface energy should also depend on $\eta_{\alpha}$, because a new surface appears due to the exit of dislocations and the production of steps, and surface energy is proportional to the number of exiting dislocations and depends on the orientation of the new area. At the same time dislocation core energy disappears. While an explicit expression for $\gamma_{0 s}$ requires a separate study, which is beyond the goals of the current paper, in the simplest case we can assume $\gamma_{0 s}\left(\eta_{\alpha}\right)=$ const $+P\left(\boldsymbol{n}_{0}^{\alpha}\right) \eta_{\alpha}$, where $P\left(\boldsymbol{n}_{0}^{\alpha}\right)$ characterizes energetic changes associated with the exit of a single dislocation at the surface. Let us elaborate

$$
\begin{aligned}
& \boldsymbol{k}_{0} \cdot \rho_{0} \frac{\partial \psi}{\partial \boldsymbol{\nabla}_{0} \eta_{\alpha}}=\boldsymbol{k}_{0} \cdot \beta\left(\boldsymbol{\nabla}_{0} \bar{\eta}_{\alpha}+\left[M\left(1-\bar{\eta}_{\alpha}\right)^{2}-1\right]\left(\boldsymbol{\nabla}_{0} \bar{\eta}_{\alpha} \cdot \boldsymbol{n}_{0}^{\alpha}\right) \boldsymbol{n}_{0}^{\alpha}\right)= \\
& \beta\left(\boldsymbol{k}_{0} \cdot \boldsymbol{m}_{0}^{\alpha} \nabla_{0}^{m} \bar{\eta}_{\alpha}+M\left(1-\bar{\eta}_{\alpha}\right)^{2} \nabla_{0}^{n} \bar{\eta}_{\alpha} \boldsymbol{k}_{0} \cdot \boldsymbol{n}_{0}^{\alpha}\right)=-\frac{\partial \gamma_{0 s}\left(\eta_{\alpha}\right)}{\partial \eta_{\alpha}} .
\end{aligned}
$$

where decomposition Eq.(70) was used. The term with $M$ disappears when dislocation completes $\left(\bar{\eta}_{\alpha} \rightarrow 1\right)$, which is expected for the stationary state. In any case, it was introduced to regularize the differential equation and can be omitted in the boundary conditions. Thus

$$
\beta \boldsymbol{k}_{0} \cdot \boldsymbol{m}_{0}^{\alpha} \nabla_{0}^{m} \bar{\eta}_{\alpha}=\beta \boldsymbol{k}_{0} \cdot \boldsymbol{m}_{0}^{\alpha} \nabla_{0} \eta_{\alpha} \cdot \boldsymbol{m}_{0}^{\alpha}=-\frac{\partial \gamma_{0 s}\left(\eta_{\alpha}\right)}{\partial \eta_{\alpha}} .
$$

In the simplest case when the surface energy is independent of $\eta_{\alpha}$ and $Q_{\alpha}^{0}=0$, one obtains

$$
\nabla_{0}^{m} \bar{\eta}_{\alpha}=\nabla_{0} \eta_{\alpha} \cdot \boldsymbol{m}_{0}^{\alpha}=0
$$

It is remarkable that Eq.(91) is independent of the direction normal to the surface. Since for the dislocation band within a volume and away from the dislocation core directions of $\nabla_{0} \eta_{\alpha}$ and $\boldsymbol{b}_{0}^{\alpha}$ are orthogonal and Eq.(91) is satisfied, the boundary condition Eq.(91) does not change the slip direction when the dislocation exits a crystal.

In order to transform the boundary conditions Eq.(88) to the actual configuration, first we 
introduce the thermodynamic forces $\boldsymbol{\Theta}_{\alpha}$ in the actual configuration via the equation

$$
\Theta_{\alpha}^{0} \cdot \boldsymbol{k}_{0} d S_{0}=\boldsymbol{\Theta}_{\alpha} \cdot \boldsymbol{k} d S
$$

where $k$ is the unit outer normal to the external surface $S$ in the actual configuration. In the phase field approach, the external surface remains smooth in the actual configuration after dislocations exit the crystal, i.e., normal $\boldsymbol{k}$ is defined unambiguously. Using Nanson's relationship between an oriented surface in the undeformed and deformed states (Lurie (1990)), $\boldsymbol{k}_{0} d S_{0}=J^{-1} \boldsymbol{F}^{T} \cdot \boldsymbol{k} d S$, and substituting it in Eq.(92), one obtains

$$
\boldsymbol{\Theta}_{\alpha}=J^{-1} \boldsymbol{F} \cdot \boldsymbol{\Theta}_{\alpha}^{0}=\rho_{0} \boldsymbol{F} \cdot \frac{\partial \psi}{\partial \boldsymbol{\nabla}_{0} \eta_{\alpha}}=\rho \frac{\partial \psi}{\partial \boldsymbol{\nabla} \eta_{\alpha}} .
$$

where Eq.(110) from Appendix was utilized for the last transformation. Combining Eqs.(88), (92), and (93), we obtain boundary conditions in $\Omega$ :

$$
\boldsymbol{k} \cdot \rho \frac{\partial \psi}{\partial \boldsymbol{\nabla} \eta_{\alpha}}=-\frac{\partial \gamma_{0 s}\left(\eta_{\alpha}\right)}{\partial \eta_{\alpha}} \frac{d S_{0}}{d S}=-\frac{\partial \gamma_{s}\left(\eta_{i}\right)}{\partial \eta_{\alpha}} .
$$

Here, the surface energy per unit deformed area, $\gamma_{s}=\gamma_{0 s} \frac{d S_{0}}{d S}$, has been introduced. Consequently, the structure of the boundary conditions in the actual and reference configurations is the same. Using Eqs. (89) and (90), and Eq.(110) from Appendix, the boundary conditions Eq.(94) can be transformed as

$$
\begin{aligned}
& \boldsymbol{k} \cdot \rho \frac{\partial \psi}{\partial \boldsymbol{\nabla} \eta_{\alpha}}=\boldsymbol{k} \cdot \rho_{0} \boldsymbol{F} \cdot \frac{\partial \psi}{\partial \nabla_{0} \eta_{\alpha}} \\
& =\frac{\rho}{\rho_{0}} \beta \boldsymbol{k} \cdot \boldsymbol{F} \cdot \boldsymbol{m}_{0}^{\alpha} \nabla_{0}^{m} \bar{\eta}_{\alpha}=\frac{1}{J} \beta \boldsymbol{k} \cdot \boldsymbol{F} \cdot \boldsymbol{m}_{0}^{\alpha} \boldsymbol{\nabla}_{0} \bar{\eta}_{\alpha} \cdot \boldsymbol{m}_{0}^{\alpha} .
\end{aligned}
$$

Then, using Eqs.(80) and (85), the boundary conditions in the actual configuration Eq.(95) can be expressed as

$$
\frac{1}{J} \beta\left(\boldsymbol{k} \cdot \boldsymbol{F} \cdot \boldsymbol{m}_{0}^{\alpha}\right) \boldsymbol{\nabla}_{0} \bar{\eta}_{\alpha} \cdot \boldsymbol{m}_{0}^{\alpha}=\frac{1}{J} \beta\left(\boldsymbol{k} \cdot \boldsymbol{F} \cdot \boldsymbol{F}_{e}^{-1} \cdot \boldsymbol{m}^{\alpha}\right) \boldsymbol{\nabla} \bar{\eta}_{\alpha} \cdot \boldsymbol{F} \cdot \boldsymbol{F}_{e}^{-1} \cdot \boldsymbol{m}^{\alpha}=-\frac{\partial \gamma_{s}\left(\eta_{i}\right)}{\partial \eta_{\alpha}} .
$$

When change in surface energy is negligible, Eq.(96) reduces to

$$
\nabla_{0} \eta_{\alpha} \cdot \boldsymbol{m}_{0}^{\alpha}=\nabla \eta_{\alpha} \cdot \boldsymbol{F} \cdot \boldsymbol{F}_{e}^{-1} \cdot \boldsymbol{m}^{\alpha}=0
$$

which is a counterpart of Eq.(91) in the actual configuration.

\section{Comparison with some previous small-strain theories}

In this section we discuss a serious shortcoming of the PFA to dislocations which appears when some of our conditions are not satisfied. In the phase field theory of dislocations of 
Rodney and Finel (2001); Wang et al. (2001a,b,c); Jin and Khachaturyan (2001); Wang et al. (2003); Kundin et al. (2011); Koslowski et al. (2002); Koslowski and Ortiz (2004); Alvarez et al. (2006); Rodney et al. (2003); Elder et al. (2002); Shen and Wang (2004); Wang and Li (2010); Hunter et al. (2010, 2011), the following expressions for the Burgers vector $\overline{\boldsymbol{b}}_{\alpha}$ (and consequently the plastic strain) and crystalline energy are accepted

$$
\overline{\boldsymbol{b}}_{\alpha}=\boldsymbol{b}_{0}^{\alpha} \eta_{\alpha} ; \quad \boldsymbol{\varepsilon}_{p}=\frac{1}{H^{\alpha}} \boldsymbol{b}_{0}^{\alpha} \otimes \boldsymbol{n}_{0}^{\alpha} \eta_{\alpha}=\gamma_{\alpha} \boldsymbol{m}_{0}^{\alpha} \otimes \boldsymbol{n}_{0}^{\alpha} \eta_{\alpha} ; \quad \rho_{0} \psi^{c}=A_{\alpha} \sin ^{2}\left(\pi \eta_{\alpha}\right) .
$$

instead of Eqs.(6) and (54) in the current paper. We limit ourselves to the single slip system here. The thermodynamic equilibrium condition Eq.(39) results in

$$
\boldsymbol{\sigma}: \gamma_{\alpha} \boldsymbol{m}_{0}^{\alpha} \otimes \boldsymbol{n}_{0}^{\alpha}=\tau_{\alpha} \gamma_{\alpha}=\pi A_{\alpha} \sin \left(2 \pi \eta_{\alpha}\right) \quad \rightarrow \quad \eta_{\alpha}=\frac{1}{2 \pi} \arcsin \left(\frac{\tau_{\alpha} \gamma_{\alpha}}{\pi A_{\alpha}}\right)+n
$$

where $n$ is the number of dislocations. Eq.(99) represents the equilibrium shear stress $\tau_{\alpha}-\eta_{\alpha}$ relationship for this model, which is plotted in Fig. 3. In contrast to our model, Eq.(99) does not possess roots $\eta_{\alpha}=n$ that are independent of stresses. Thus, for any stress $\tau_{\alpha}$ on the stable branches of the $\tau_{\alpha}-\eta_{\alpha}$ curve in Fig. 3, there is a corresponding equilibrium value of the order parameter and consequently the Burgers vector. Such stress-dependence of the Burgers vector is unphysical and is in conflict with the definition of the Burgers vector and plastic strain in the macroscopic plasticity theory. It affects stresses, their distribution, and consequently the dislocation evolution when the boundary-value problem is solved. Also, if stress oscillates $\tau_{\alpha}$ within the stable branch, the order parameter changes as well, causing dissipation when we expect reversible elastic behavior.

The stress-dependence of the Burgers vector was eliminated in $\mathrm{Hu}$ et al. (2004) using the following equations:

$$
\overline{\boldsymbol{b}}_{\alpha}=\boldsymbol{b}_{0}^{\alpha} \chi\left(\eta_{\alpha}\right) ; \quad \boldsymbol{\varepsilon}_{p}=\frac{1}{H^{\alpha}} \boldsymbol{b}_{0}^{\alpha} \otimes \boldsymbol{n}_{0}^{\alpha} \chi\left(\eta_{\alpha}\right)=\gamma_{\alpha} \boldsymbol{m}_{0}^{\alpha} \otimes \boldsymbol{n}_{0}^{\alpha} \chi\left(\eta_{\alpha}\right) ; \quad \chi\left(\eta_{\alpha}\right)=\eta_{\alpha}-\frac{1}{2 \pi} \sin \left(2 \pi \eta_{\alpha}\right) .(100)
$$

and the same crystalline energy Eq.(98). Function $\chi\left(\eta_{\alpha}\right)$ satisfies condition (40), i.e., it has zero derivative for integer $\eta_{\alpha}$. The thermodynamic equilibrium condition Eq.(39) results in

$$
\tau_{\alpha} \gamma_{\alpha} \sin ^{2}\left(\pi \eta_{\alpha}\right)=\pi A_{\alpha} \sin \left(\pi \eta_{\alpha}\right) \cos \left(\pi \eta_{\alpha}\right)
$$

One of the solutions of Eq.(101) is $\sin \left(\pi \eta_{\alpha}\right)=0$ and consequently $\eta_{\alpha}=n$, as desired. Another root results in the $\tau_{\alpha}-\eta_{\alpha}$ curve (Fig. 3):

$$
\tau_{\alpha} \gamma_{\alpha}=\pi A_{\alpha} \cot \left(\pi \eta_{\alpha}\right)
$$




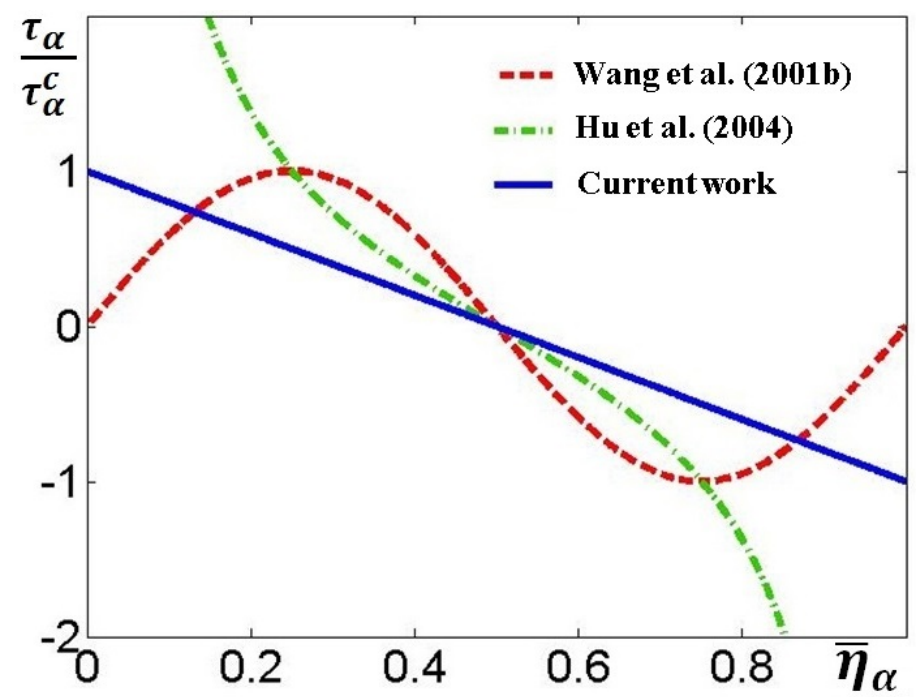

Figure 3: The normalized equilibrium resolved shear stress $\frac{\tau_{\alpha}}{\tau_{\alpha}^{c}}-\bar{\eta}_{\alpha}$ curves of the current model and those of Wang et al. (2001b) and Hu et al. (2004). For the model of Hu et al. (2004), $\tau_{\alpha}^{c}=\frac{\pi A_{\alpha}}{\gamma_{\alpha}}$, similar to that of Wang et al. (2001b).

Apparently, Eq.(102) requires an infinite magnitude of stresses at $\eta_{\alpha}=n$ to initiate lattice

instability and nucleate dislocation. This is because $\frac{d^{2} \chi\left(\eta_{\alpha}=n_{\alpha}\right)}{d \eta_{\alpha}^{2}}=0$ and the left side of the instability criterion (49) is identically zero, while the right side is finite. Thus, with such a theory, to nucleate each next dislocation, one needs either a strong noise due to thermal fluctuations or a numerical error leading to a deviation of $\eta_{\alpha}$ from $n$.

For comparison, the normalized equilibrium $\tau_{\alpha}-\eta_{\alpha}$ curve for our theory (Eq.(63)) is also plotted in Fig. 3. Instability starts when the magnitude of stress exceeds some critical value and $\eta_{\alpha}$ evolves to the nearest integer value. Stress oscillation below critical value keeps $\bar{\eta}_{\alpha}=0$ and does not cause an artificial dissipation. The stable equilibrium value of $\eta_{\alpha}=n$, which along with the equilibrium magnitude of the Burgers vector are independent of stresses. Thus, our theory does not have contradictions typical of previous approaches.

\section{Concluding remarks}

In this paper, a thermodynamically consistent large strain phase field approach to dislocations at the nanoscale is developed. It includes kinematics consistent with phenomenological crystal plasticity. Thermodynamic treatments include a traditional application of the first and second laws of thermodynamics and additional conditions related to thermodynamic equilibrium and its instability, similar to those formulated earlier for phase transformations (Levitas 
and Preston (2002a,b); Levitas et al. (2003); Levitas (2013b)). The theory incorporates explicitly the dislocation height and regularizing contribution to the gradient energy, which excludes the localization of a dislocation within a band smaller than the prescribed height (i.e., makes formulation well-posed) but does not produce artificial interface energy. An additional energy term is introduced that penalizes the interaction of different dislocations at the same point. Complete system of equations consists of kinematic equations (1), (2), (10), and (62) for large strains or equations (3), (5), (17) and (62) for small strains; equations (58) and (59) for stresses; equations (53)-(57) and (65) for free energy; Ginzburg-Landau equations (72) and (74) in the reference and actual configurations in the compact form, or equations (79) and (84) in the detailed form, or equation (86) for small strains; expressions for resolved shear stress (35) and (37); momentum balance equation, and boundary conditions for order parameters in the reference (Eq. 90) or in the deformed (Eq. 96) configurations. Numerical simulations based on the developed theory are presented in Levitas and Javanbakht (2012) and in a paper currently in progress by Javanbakht and Levitas (2014).

The developed approach requires a fine FEM discretization typical of the PFA to any phenomena. That is why it cannot be applied for large scale problems and is effective when short range interactions of dislocations and dislocations and other defects such as point defects, grain and twin boundaries, and phase interfaces are of importance. One of our main interests is generalizing the developed theory for the interaction between dislocations and phase transformations, and the first results in this direction are already obtained (Levitas and Javanbakht (2013, 2014)). They include both mutual assistance and competition between phase transformation and dislocation evolution. Dislocations create the stress concentration required for the nucleation of a product phase but produce a semicoherent interface and athermal resistance to interface motion. Phase transformation can partially or completely substitute dislocations as a mechanism of plastic deformation and stress relaxation but can also generate stresses required for nucleation of dislocations. At the microscale, such an interaction has been studied analytically and numerically by Levitas et al. (1998); Levitas (2000); Idesman et al. (2000). A similar interaction occurs between dislocations and twins.

Our theory could be generalized in order to take into account the line tension of a dislocation, similar to the surface tension (stresses) for a phase interface (Levitas and Javanbakht 
(2011b); Levitas and Samani (2011b); Levitas (2013a,c, 2014)). Generalization for partial dislocations and dislocation reactions can be done using approaches in Shen and Wang (2004); $\mathrm{Hu}$ et al. (2004); Wang and $\mathrm{Li}$ (2010) but with an additional requirements formulation in the current paper. Also, dislocation evolution can be coupled to the diffusion of species utilizing the Cahn-Hilliard equation for conserved order parameters (Hu et al. (2004)).

\section{Acknowledgement}

The support of NSF (CMMI-0969143), ARO (W911NF-12-1-0340), DARPA (W31P4Q-131-0010), ONR (N00014-12-1-0525), and Iowa State University are gratefully acknowledged. 


\section{References}

\section{References}

Abu Al-Rub, R. K., Voyiadjis, G. Z., 2005. A direct finite element implementation of the gradient-dependent theory. Int. J. Numer. Meth. Engng. 63, 603-629.

Alvarez, O., Hoch, P., Le Bouar, Y., Monneau, R., 2006. Dislocation dynamics: short-time existence and uniqueness of the solution. Arch. Ration. Mech. Anal. 181, 449-504.

Artemev, A., Jin, Y., Khachaturyan, A. G., 2001. Three-dimensional phase field model of proper martensitic transformation. Acta Mater. 49, 1165-1177.

Asaro, R. J., 1983. Crystal plasticity. J. Appl. Mech. Trans. ASME. 50, 921-934.

Chen, L. Q., 2002. Phase-field models for microstructure evolution. Annu. Rev. Mater. Res. $32,113-140$.

Clayton, J. D., Knap, J., 2011. A phase field model of deformation twinning: nonlinear theory and numerical simulations. Physica D-Nonlinear Phenomena. 240, 841-858.

Cui, Y. N., Lin, P., Liu, Z. L., Zhuang, Z., 2014. Theoretical and numerical investigations of single arm dislocation source controlled plastic flow in FCC micropillars. Int. J. Plast. 55, 279-292.

Elder, K. R., Katakowski, M., Haataja, M., Grant, M., 2002. Modeling elasticity in crystal growth. Phys. Rev. Lett. 88, 245701.

Engels, P., Ma, A., Hartmaier, A., 2012. Continuum simulation of the evolution of dislocation densities during nanoindentation. Int. J. Plast. 38, 159-169.

Fan, J., Stewart, R. J., Zeng, X., 2011. A multiscale method for dislocation nucleation and seamlessly passing scale boundaries. Int. J. Plast. 27, 2103-2124.

Havner, K. S., 1992. Finite plastic deformation of crystalline solids. Cambridge Monographs on Mechanics and Applied Mathematics. Press Syndicate of the University of Cambridge, New York. 
Hirth, J. P., Lothe, J., 1992. Theory of dislocations. Krieger Publishing Company, Malabar, Florida.

Hu, S. Y., Chen, L. Q., 2001. Solute segregation and coherent nucleation and growth near a dislocation - a phase-field model integrating defect and phase microstructures. Acta. Mater. $49,463-472$.

Hu, S. Y., Chen, L. Q., 2002. Diffuse-interface modeling of composition evolution in the presence of structural defects. Comput. Mater. Sci. 23, 270-282.

Hu, S. Y., Li, Y. L., Zheng, Y. X., Chen, L. Q., 2004. Effect of solutes on dislocation motion a phase-field simulation. Int. J. Plast. 20, 403-425.

Huang, M., Zhao, L., Tong, J., 2012. Discrete dislocation dynamics modelling of mechanical deformation of nickel-based single crystal superalloys. Int. J. Plast. 28, 141-158.

Hunter, A., Beyerlein, I. J., Germann, T. C., Koslowski, M., 2011. Influence of the stacking fault energy surface on partial dislocations in fcc metals with a three-dimensional phase field dislocations dynamics model. Phys. Rev. B. 84, 144108.

Hunter, A., Le, C., Saied, F., Koslowski, M., 2010. Large-scale 3D phase field dislocation dynamics simulations on high-performance architectures. Int. J. High Perform. Comput. Appl. 25, 223-235.

Idesman, A. V., Levitas, V. I., Stein, E., 2000. Structural changes in elastoplastic materials: a unified finite element approach for phase transformation, twinning and fracture. Int. J. Plast. 16, 893-949.

Javanbakht, M., Levitas, V. I., 2014. Phase field simulations of dislocation evolution at large strains (in preparation).

Jin, Y. M., Artemev, A., Khachaturyan, A. G., 2001. Three-dimensional phase field model of low-symmetry martensitic transformation in polycrystal: Simulation of $\zeta_{2}^{\prime}$ martensite in AuCd alloys. Acta Mater. 49, 2309-2320.

Koslowski, M., 2007. Scaling laws in plastic deformation. Phil. Mag. 87, 1175-1184. 
Jin, Y. M., Khachaturyan, A. G., 2001. Phase field microelasticity theory of dislocation dynamics in a polycrystal: model and three-dimensional simulations. Philos. Mag. 81, 607-616.

Koslowski, M., Cuitino, M., Ortiz, A. M., 2002. A phase-field theory of dislocation dynamics, strain hardening and hysteresis in ductile single crystals. J. Mech. Phys. Solids. 50, 25972635.

Koslowski, M., Ortiz, A. M., 2004. A multi-phase field model of planar dislocation networks. Model. Simul. Mater. Sci. Eng. 12, 1087-1097.

Kundin, J., Emmerich, H., Zimmer, J., 2011. Mathematical concepts for the micromechanical modelling of dislocation dynamics with a phase-field approach. Philos. Mag. 91, 97-121.

Lee, D.-W., Kim, H., Strachan, A., Koslowski, M., 2011. Effect of core energy on mobility in a continuum dislocation model. Phys. Rev. B. 83, 104101.

Lei, L., Koslowski, M., 2011. Mesoscale modeling of dislocations in molecular crystals. Philos. Mag. 91, 865-878.

Levitas, V. I., 1996. Large deformation of materials with complex rheological properties at normal and high pressure. Nova Science Publishers, New York.

Levitas, V. I., 2000. Structural changes without stable intermediate state in inelastic material. part II. Applications to displacive and diffusional-displacive phase transformations, straininduced chemical reactions and ductile fracture. Int. J. Plast. 16, 851-892.

Levitas, V. I., 2013a. Interface stress for nonequilibrium microstructures in the phase field approach: exact analytical results. Phys. Rev. B. 87, 054112.

Levitas, V. I., 2013b. Phase-field theory for martensitic phase transformations at large strains. Int. J. Plast. 49, 85-118.

Levitas, V. I., 2013c. Thermodynamically consistent phase field approach to phase transformations with interface stresses. Acta Mater. 61, 4305-4319.

Levitas, V. I., 2014b. Phase field approach to martensitic phase transformations with large strains and interface stresses. J. Mech. Phys. Solids. 70, 154-189. 
Levitas, V. I., Idesman, A. V., Lee, D.-W., 2010. Interface propagation and microstructure evolution in phase field models of stress-induced martensitic phase transformations. Int. J. Plast. 25, 395-422.

Levitas, V. I., Idesman, A. V., Stein, E., 1998. Finite element simulation of martensitic phase transitions in elastoplastic materials. Int. J. Solids. Struct. 35, 855-887.

Levitas, V. I., Javanbakht, M., 2010. Surface tension and energy in multivariant martensitic transformations: phase-field theory, simulations, and model of coherent interface. Phys. Rev. Lett. 105, 165701.

Levitas, V. I., Javanbakht, M., 2011a. Phase-field approach to martensitic phase transformations: effect of martensite-martensite interface energy. Int. J. Mat. Res. 102(6), 652-665.

Levitas, V. I., Javanbakht, M., 2011b. Surface-induced phase transformations: multiple scale and mechanics effects and morphological transitions. Phys. Rev. Lett. 107, 175701.

Levitas, V. I., Javanbakht, M., 2012. Advanced phase-field approach to dislocation evolution. Phys. Rev. B Rapid Communications 86, 140101.

Levitas, V. I., Javanbakht, M., 2013. Phase field approach to interaction of phase transformation and dislocation evolution. Appl. Phys. Lett. 102, 251904.

Levitas, V. I., Javanbakht, M., 2014. Phase transformations in nanograin materials under high pressure and plastic shear: nanoscale mechanisms. Nanoscale 6, 162-166.

Levitas V. I., Lee D.-W., 2007. Athermal resistance to an interface motion in phase field theory of microstructure evolution. Phys. Rev. Lett. 99, 245701.

Levitas, V. I., Levin, V. A., Zingerman, K. M., Freiman, E. I., 2009. Displacive phase transitions at large strains: phase-field theory and simulations. Phys. Rev. Lett. 103, 025702.

Levitas, V. I., Preston, D., L., 2002a. Three-dimensional Landau theory for multivariant stress-induced martensitic phase transformations. I. Austenite-martensite. Phys. Rev. B. $66,134206$. 
Levitas, V. I., Preston, D., L., 2002b. Three-dimensional Landau theory for multivariant stressinduced martensitic phase transformations. II. Multivariant phase transformations and stress space analysis. Phys. Rev. B. 66, 134207.

Levitas, V. I., Preston, D. L., 2005. Thermomechanical lattice instability and phase field theory of martensitic phase transformations, twinning and dislocations at large strains. Phys. Lett. A. $343,32-39$.

Levitas, V. I., Preston, D. L., Lee, D.-W., 2003. Three-dimensional Landau theory for multivariant stress-induced martensitic phase transformations. III. Alternative potentials, critical nuclei, kink solutions, and dislocation theory. Phys. Rev. B. 68, 134201.

Levitas, V. I., Samani, K., 2011a. Coherent solid-liquid interface with stress relaxation in a phase-field approach to the melting/freezing transition. Phys. Rev. B. 84, 140103.

Levitas, V. I., Samani, K., 2011b. Size and mechanics effects in surface-induced melting of nanoparticles. Nat. Commun. 2, 284.

Lipowsky, R., 1982. Critical surface phenomena at first-order bulk transitions. Phys. Rev. Lett. $49,1575-1578$.

Li., D., Zbib, H., Sun, X., Khaleel, M., 2014. Predicting plastic flow and irradiation hardening of iron single crystal with mechanism-based continuum dislocation dynamics. Int. J. Plast. $52,3-17$.

Liu, Z. L., Zhuang, Z., Liu, X. M., Zhao, X. C., Zhang, Z. H., 2011. A dislocation dynamics based higher-order crystal plasticity model and applications on confined thin-film plasticity. Int. J. Plast. 27, 201-216.

Lubarda, V. A., 2002. ELastoplasticity theory. CRC Press, Boca Raton.

Lurie, A. I., 1990. Non-linear theory of elasticity. North-Holland Series in Applied Mathematics and Mechanics.

Malvern, L.E., 1977. Introduction to the mechanics of a continuous medium. Prentice Hall, N.J. 
Nabarro, F. R. N., 1947. Dislocations in a simple cubic lattice. Proc. Phys. Soc. 59, 256-272.

Nabarro, F. R. N., 1951. The synthesis of elastic dislocation fields. Philos. Mag. 42, 1224.

Öztop, M. S., Niordson, C. F., Kysar, J. W., 2013. Length-scale effect due to periodic variation of geometrically necessary dislocation densities. Int. J. Plast. 41, 189-201.

Peierls, R., 1940. The size of a dislocation. Proc. Phys. Soc. 52, 34.

Pluis, B., Frenkel, D., Van der Veen, J. F., 1990. Surface-induced melting and freezing II. A semi-empirical Landau-type model. Surf. Sci. 239, 282-300.

Porter, D. A., Easterling, K. E., 1992. Phase transformations in metals and alloys. Chapman \& Hall, London, England.

Rodney, D., Finel, A., 2001. Phase field methods and dislocations. MRS Symp. Proc. 652, 491.

Rodney, D., Le Bouar, Y., Finel, A., 2003. Phase field methods and dislocations. Acta Mater. 51, 17-30.

Shen, C., Wang, Y., 2004. Incorporation of $\gamma$-surface to phase field model of dislocations: simulating dislocation dissociation in fcc crystals. Acta Mater. 52, 683-691.

Vorontsov, V. A., Shen, C., Wang, Y., Dye, D., Rae, C. M. F. 2010. Shearing of $\gamma^{\prime}$ precipitates by $a<112>$ dislocation ribbons in Ni-base superalloys: a phase field approach. Acta Mater., 58, 2010, 4110-4119.

Wang, Y. U., Jin, Y. M., Cuitino, A. M., Khachaturyan, A. G., 2001a. Application of phase field microelasticity theory of phase transformations to dislocation dynamics: model and three-dimensional simulations in a single crystal. Philos. Mag. 81, 385-393.

Wang, Y. U., Jin, Y. M., Cuitino, A. M., Khachaturyan, A. G., 2001b. Nanoscale phase field microelasticity theory of dislocations: model and 3D simulations. Acta Mater. 49, 1847-1857.

Wang, Y. U., Jin, Y. M., Cuitino, A. M., Khachaturyan, A. G., 2001c. Phase field microelasticity theory and modeling of multiple dislocation dynamics. Appl. Phys. Lett. 78, 2324-2326. 
Wang, Y. U., Jin, Y. M., Khachaturyan, A. G., 2003. Phase field microelasticity modeling of dislocation dynamics near free surface and in heteroepitaxial thin films. Acta. Mater. 51, 4209-4223.

Wang, Y., Khachaturyan, A. G., 1997. Three-dimensional field model and computer modeling of martensitic transformations. Acta Mater. 45, 759-773.

Wang, Y. U., Li, J., 2010. Phase field modeling of defects and deformation. Acta. Mater. 58, 1212-1235.

Wang, Y., Shen, C., 2003. Phase field model of dislocation networks. 51, 2595-2610.

Wang, G., Strachan, A., Cagin, T., Goddard III, W. A., 2001. Molecular dynamics simulations of $1 / 2 \mathrm{a}<111>$ screw dislocation in Ta. Mater. Sci. Eng. A. 309-310, 133-137.

Xiong, L., McDowell, D. L., Chen, Y., 2014. Sub-THz phonon drag on dislocations by coarsegrained atomistic simulations. Int. J. Plast. 55, 268-278.

Yanilkin, A. V., Krasnikov, V. S., Kuksin, A. Y., Mayer, A. E., 2014. Dynamics and kinetics of dislocations in $\mathrm{Al}$ and $\mathrm{AlCu}$ alloy under dynamic loading. Int. J. Plast., 55, 94-107.

Zbib, H. M., Aifantis, E. C., 1992. On the gradient-dependent theory of plasticity and shear banding. Acta Mech. 92, 209-225.

Zhou, N., Shen, C., Mills, M. J., Wang, Y., 2007. Phase field modeling of channel dislocation activity and $\gamma^{\prime}$ rafting in single crystal Ni-Al. Acta Mater. 55, 5369-81. 


\section{Appendix. Some derivations}

1. We will prove that for a tensor $\boldsymbol{A}$ of an arbitrary rank the following identity is valid:

$$
\boldsymbol{\nabla} \cdot\left(\rho_{0} \boldsymbol{A}\right)=J \nabla \cdot(\rho \boldsymbol{A})=\frac{\rho_{0}}{\rho} \nabla \cdot(\rho \boldsymbol{A}) .
$$

We will start with

$$
\frac{\partial\left(\rho_{0} A_{i j \ldots k}\right)}{\partial r_{k}}=\frac{\partial\left(\rho J A_{i j \ldots k}\right)}{\partial r_{k}}=J \frac{\partial\left(\rho A_{i j \ldots k}\right)}{\partial r_{k}}+\rho A_{i j \ldots k} \frac{\partial J}{\partial r_{k}} .
$$

Then

$$
\frac{\partial J}{\partial r_{k}}=\frac{\partial J}{\partial F_{m n}} \frac{\partial F_{m n}}{\partial r_{k}} ; \quad \frac{\partial F_{m n}}{\partial r_{k}}=\frac{\partial}{\partial r_{k}} \frac{\partial r_{m}}{\partial r_{0 n}}=\frac{\partial}{\partial r_{0 n}} \frac{\partial r_{m}}{\partial r_{k}}=\frac{\partial \delta_{m k}}{\partial r_{0 n}}=0 ; \quad \nabla J=0 .
$$

Thus, for gradient in the current configuration, the Jacobian $J$ can be treated as constant. Eq.(104) simplifies to

$$
\frac{\partial\left(\rho_{0} A_{i j \ldots k}\right)}{\partial r_{k}}=J \frac{\partial\left(\rho A_{i j \ldots k}\right)}{\partial r_{k}} .
$$

which is a component form of Eq.(103).

2. We will prove the following identity:

$$
\frac{1}{\rho_{0}} \nabla_{0} \cdot\left(\rho_{0} \frac{\partial \psi}{\partial \nabla_{0} \eta}\right)=\frac{1}{\rho} \nabla \cdot\left(\rho \frac{\partial \psi}{\partial \nabla \eta}\right) .
$$

In the component form

$$
\nabla_{0} \cdot\left(\rho_{0} \frac{\partial \psi}{\partial \nabla_{0} \eta}\right):=\nabla_{0}^{k}\left(\rho_{0} \frac{\partial \psi}{\partial \nabla_{0}^{k} \eta}\right)
$$

Then,

$$
\frac{\partial \psi}{\partial \nabla_{0}^{k} \eta}=\frac{\partial \psi}{\partial \nabla^{m} \eta} \frac{\partial \nabla^{m} \eta}{\partial \nabla_{0}^{k} \eta}=\frac{\partial \psi}{\partial \nabla^{m} \eta} F_{k m}^{-1}=\frac{\partial \psi}{\partial \nabla^{m} \eta} \frac{\partial r_{0 k}}{\partial r_{m}} .
$$

which can be presented in index-free notations like

$$
\frac{\partial \psi}{\partial \boldsymbol{\nabla}_{0} \eta}=\boldsymbol{F}^{-1} \cdot \frac{\partial \psi}{\partial \boldsymbol{\nabla} \eta} ; \quad \frac{\partial \psi}{\partial \boldsymbol{\nabla} \eta}=\boldsymbol{F} \cdot \frac{\partial \psi}{\partial \boldsymbol{\nabla}_{0} \eta} .
$$

After substituting Eq.(109) in Eq.(108), we obtain

$$
\begin{aligned}
& \frac{1}{\rho_{0}} \nabla_{0}^{k}\left(\rho_{0} \frac{\partial \psi}{\partial \nabla_{0}^{k} \eta}\right)=\frac{1}{\rho_{0}} \frac{\partial}{\partial r_{0 k}}\left(\rho_{0} \frac{\partial \psi}{\partial \nabla^{m} \eta} \frac{\partial r_{0 k}}{\partial r_{m}}\right) \\
& =\frac{1}{\rho_{0}}\left(\frac{\partial}{\partial r_{0 k}}\left(\rho_{0} \frac{\partial \psi}{\partial \nabla^{m} \eta}\right) \frac{\partial r_{0 k}}{\partial r_{m}}+\rho_{0} \frac{\partial \psi}{\partial \nabla^{m} \eta} \frac{\partial}{\partial r_{m}} \frac{\partial r_{0 k}}{\partial r_{0 k}}\right)=\frac{1}{\rho_{0}} \frac{\partial}{\partial r_{m}}\left(\rho_{0} \frac{\partial \psi}{\partial \nabla^{m} \eta}\right) \\
& =\frac{1}{\rho_{0}} \nabla \cdot\left(\rho_{0} \frac{\partial \psi}{\partial \boldsymbol{\nabla} \eta}\right)=\frac{J}{\rho_{0}} \nabla \cdot\left(\rho \frac{\partial \psi}{\partial \nabla \eta}\right)=\frac{1}{\rho} \boldsymbol{\nabla} \cdot\left(\rho \frac{\partial \psi}{\partial \nabla \eta}\right) .
\end{aligned}
$$


which proves Eq.(107). We used Eq.(103) in the last line.

2. We will prove that $\boldsymbol{\nabla}_{0} \cdot \boldsymbol{\nabla}_{0} \bar{\eta}_{\alpha}=\left(\boldsymbol{F}^{T} \cdot \boldsymbol{\nabla}\right) \cdot\left(\boldsymbol{\nabla} \bar{\eta}_{\alpha} \cdot \boldsymbol{F}\right)=\left(\boldsymbol{F} \cdot \boldsymbol{F}^{T}\right): \nabla \boldsymbol{\nabla} \bar{\eta}_{\alpha}$. Indeed

$$
\begin{aligned}
& \boldsymbol{\nabla}_{0} \cdot \boldsymbol{\nabla}_{0} \bar{\eta}_{\alpha}=\left(\boldsymbol{F}^{T} \cdot \boldsymbol{\nabla}\right) \cdot\left(\boldsymbol{\nabla} \bar{\eta}_{\alpha} \cdot \boldsymbol{F}\right)=\frac{\partial r_{b}}{\partial r_{0 k}} \frac{\partial}{\partial r_{b}}\left(\frac{\partial \bar{\eta}_{\alpha}}{\partial r_{l}} \frac{\partial r_{l}}{\partial r_{0 m}}\right) \\
& =\frac{\partial r_{b}}{\partial r_{0 k}}\left(\frac{\partial}{\partial r_{b}}\left(\frac{\partial \bar{\eta}_{\alpha}}{\partial r_{l}}\right) \frac{\partial r_{l}}{\partial r_{0 m}}+\frac{\partial \bar{\eta}_{\alpha}}{\partial r_{l}} \frac{\partial}{\partial r_{b}} \frac{\partial r_{l}}{\partial r_{0 m}}\right)=\frac{\partial r_{b}}{\partial r_{0 k}}\left(\frac{\partial}{\partial r_{b}}\left(\frac{\partial \bar{\eta}_{\alpha}}{\partial r_{l}}\right) \frac{\partial r_{l}}{\partial r_{0 m}}+\frac{\partial \bar{\eta}_{\alpha}}{\partial r_{l}} \frac{\partial}{\partial r_{0 m}} \frac{\partial r_{l}}{\partial r_{b}}\right) \\
& =\frac{\partial r_{b}}{\partial r_{0 k}} \frac{\partial}{\partial r_{b}}\left(\frac{\partial \bar{\eta}_{\alpha}}{\partial r_{l}}\right) \frac{\partial r_{l}}{\partial r_{0 m}}=\boldsymbol{F}^{T} \cdot \nabla \nabla \bar{\eta}_{\alpha} \cdot \boldsymbol{F}=\left(\boldsymbol{F} \cdot \boldsymbol{F}^{T}\right): \nabla \nabla \bar{\eta}_{\alpha} .
\end{aligned}
$$

They key point of this proof is that

$$
(\boldsymbol{\nabla} \boldsymbol{F})_{b l m}=\frac{\partial}{\partial r_{b}} \frac{\partial r_{l}}{\partial r_{0 m}}=\frac{\partial}{\partial r_{0 m}} \frac{\partial r_{l}}{\partial r_{b}}=\frac{\partial \delta_{l b}}{\partial r_{0 m}}=0 .
$$

i.e., for the gradient in the current configuration, the deformation gradient $\boldsymbol{F}$ can be treated as a constant tensor.

4. Let us prove the identity

$$
\left(\boldsymbol{n}^{\alpha} \cdot \boldsymbol{\nabla}\right)\left(\boldsymbol{\nabla} \bar{\eta}_{\alpha} \cdot \boldsymbol{F} \cdot \boldsymbol{F}_{e}^{T} \cdot \boldsymbol{n}^{\alpha}\right)=n_{i}^{\alpha} \frac{\partial^{2} \bar{\eta}_{\alpha}}{\partial r_{i} \partial r_{k}} F_{b k} F_{e}^{k b}=\boldsymbol{n}^{\alpha} \cdot \nabla \nabla \bar{\eta}_{\alpha} \cdot \boldsymbol{F} \cdot \boldsymbol{F}_{e}^{T} \cdot \boldsymbol{n}^{\alpha} .
$$

Indeed,

$$
\begin{aligned}
& \left(\boldsymbol{n}^{\alpha} \cdot \boldsymbol{\nabla}\right)\left(\boldsymbol{\nabla} \bar{\eta}_{\alpha} \cdot \boldsymbol{F} \cdot \boldsymbol{F}^{T} e_{e} \cdot \boldsymbol{n}^{\alpha}\right)=n_{i}^{\alpha} \frac{\partial}{\partial r_{i}}\left(\frac{\partial \bar{\eta}_{\alpha}}{\partial r_{k}} \frac{\partial r_{b}}{\partial r_{0 k}} \frac{\partial r_{k}}{\partial p_{b}}\right) n_{k}^{\alpha} \\
& =n_{i}^{\alpha}\left(\frac{\partial}{\partial r_{i}}\left(\frac{\partial \bar{\eta}_{\alpha}}{\partial r_{k}}\right)\left(\frac{\partial r_{b}}{\partial r_{0 k}} \frac{\partial r_{k}}{\partial p_{b}}\right)+\frac{\partial \bar{\eta}_{\alpha}}{\partial r_{k}} \frac{\partial}{\partial r_{i}}\left(\frac{\partial r_{b}}{\partial r_{0 k}} \frac{\partial r_{k}}{\partial p_{b}}\right)\right) n_{k}^{\alpha} \\
& =n_{i}^{\alpha}\left(\frac{\partial}{\partial r_{i}}\left(\frac{\partial \bar{\eta}_{\alpha}}{\partial r_{k}}\right)\left(\frac{\partial r_{b}}{\partial r_{0 k}} \frac{\partial r_{k}}{\partial p_{b}}\right)+\frac{\partial \bar{\eta}_{\alpha}}{\partial r_{k}}\left[\frac{\partial}{\partial r_{0 k}}\left(\frac{\partial r_{b}}{\partial r_{i}}\right) \frac{\partial r_{k}}{\partial p_{b}}+\frac{\partial r_{b}}{\partial r_{0 k}} \frac{\partial}{\partial p_{b}}\left(\frac{\partial r_{k}}{\partial r_{i}}\right)\right]\right) n_{k}^{\alpha} \\
& =n_{i}^{\alpha} \frac{\partial}{\partial r_{i}}\left(\frac{\partial \bar{\eta}_{\alpha}}{\partial r_{k}}\right)\left(\frac{\partial r_{b}}{\partial r_{0 k}} \frac{\partial r_{k}}{\partial p_{b}}\right) n_{k}^{\alpha}=\boldsymbol{n}^{\alpha} \cdot \nabla \nabla \bar{\eta}_{\alpha} \cdot \boldsymbol{F} \cdot \boldsymbol{F}^{T} \cdot \boldsymbol{n}^{\alpha} .
\end{aligned}
$$

Obtained results mean that for the gradient in the current configuration, not only the deformation gradient $\boldsymbol{F}$ but also elastic part $\boldsymbol{F}_{e}$ of the deformation gradient can be treated as a constant tensor. 